Supporting Information

\title{
Discovery, optimization and structure-activity relationship study of novel and potent RSK4 inhibitors as promising agents for the treatment of esophageal squamous cell carcinoma
}

Yuan Yuan ${ }^{1 \S}$, Junpeng $\mathrm{Xu}^{2 \S}$, Lei Jiang ${ }^{1 \S}$, Kangjie $\mathrm{Yu}^{2 \S}$, Yuanyuan $\mathrm{Ge}^{1}$, Mingyang $\mathrm{Li}^{2}$, Huan He ${ }^{1}$, Qiqi Niu ${ }^{1}$, Xiayu Shi ${ }^{1}$, Linni Fan², Zhuo Chen ${ }^{1}$, Zhenjiang Zhao ${ }^{1}$, Shiliang $\mathrm{Li}^{1 *}$, Yufang $\mathrm{Xu}^{1 *}$, Zhe Wang ${ }^{2 *}$, Honglin $\mathrm{Li}^{1,3 *}$

${ }^{1}$ Shanghai Key Laboratory of New Drug Design, State Key Laboratory of Bioreactor Engineering, School of Pharmacy, East China University of Science \& Technology, Shanghai, China, 200237.

${ }^{2}$ State Key Laboratory of Cancer Biology, Department of Pathology, Xijing Hospital and School of Basic Medicine, Fourth Military Medical University, Xi'an, China, 710032.

${ }^{3}$ Research and Development Department, Jiangzhong Pharmaceutical Co., Ltd., Nanchang, China, 330096.

\$Authors contributed equally to this work.

* To whom correspondence should be addressed. Email: slli403@163.com, yfxu@ecust.edu.cn, zhwang@fmmu.edu.cn, hlli@ecust.edu.cn 


\section{Contents}

Figure S1. Homology modeling of RSK4 NTKD...................................................

Figure S2. In vitro antiproliferation assay at $10 \mu \mathrm{M}$ for representative compounds ..S4

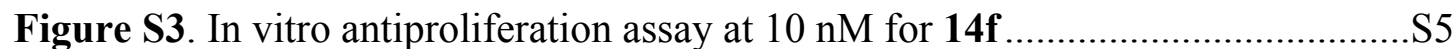

Figure S4. 14f inhibited the phosphorylation of RPS6 and GSK3 $\beta$ in tumor samples

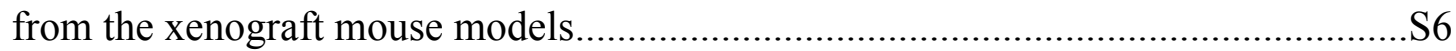

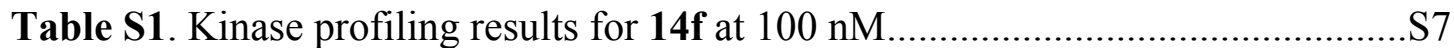

Table S2. Predicted lipophilicity and permeability properties of representative

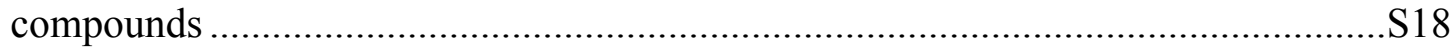

Table S3. Pharmacokinetics profiles of $\mathbf{1 4 f}$ in rats ................................................. 19

The spectra of ${ }^{1} \mathrm{H}$ NMR, ${ }^{13} \mathrm{C}$ NMR, HRMS (ESI) of all compounds and HPLC of

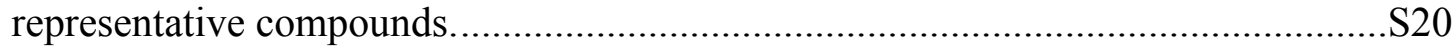



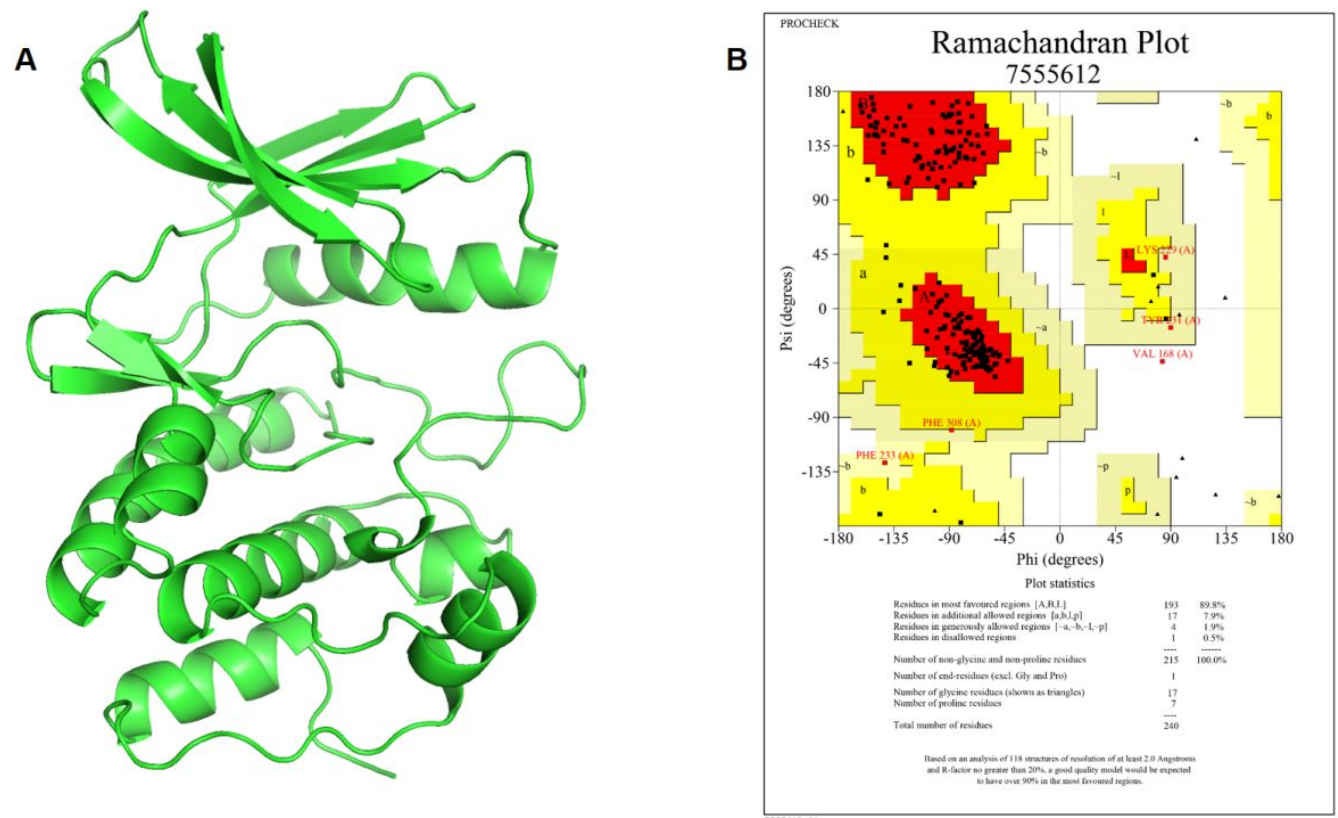

Figure S1. Homology modeling of RSK4 NTKD. (A) The modeled RSK4 NTKD structure. (B) The Ramachandran plot result of the initial RSK4 NTKD model. RSK4 NTKD comprises two lobes and a cleft, which is like all kinase. For the Ramachandran plot, residues in most favored regions are $89.8 \%$, residues in additional allowed regions are $7.9 \%$, and residues in generously allowed regions are $1.9 \%$, and the sum is $99.6 \%$, which indicates that the dihedral angle of the protein backbone is more than $95 \%$ within the allowable range. The result of Verify-3D is $89.17 \%$, which shows that the amino acid side chain conformation is reasonable. 


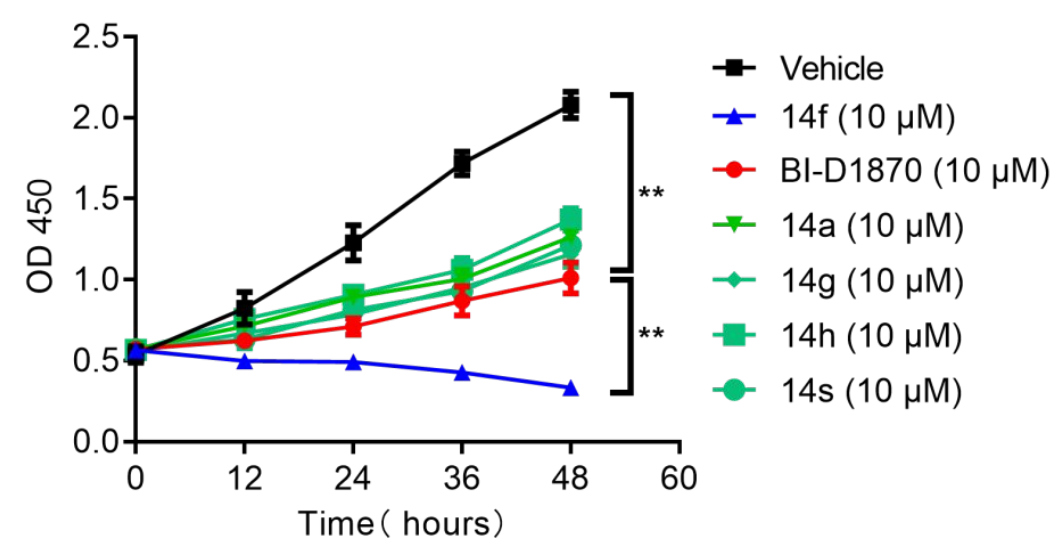

Figure S2. In vitro antiproliferation assay at $10 \mu \mathrm{M}$ for representative compounds.

$\mathbf{1 4 f}$ displayed the best antiproliferation activities among the compounds. 


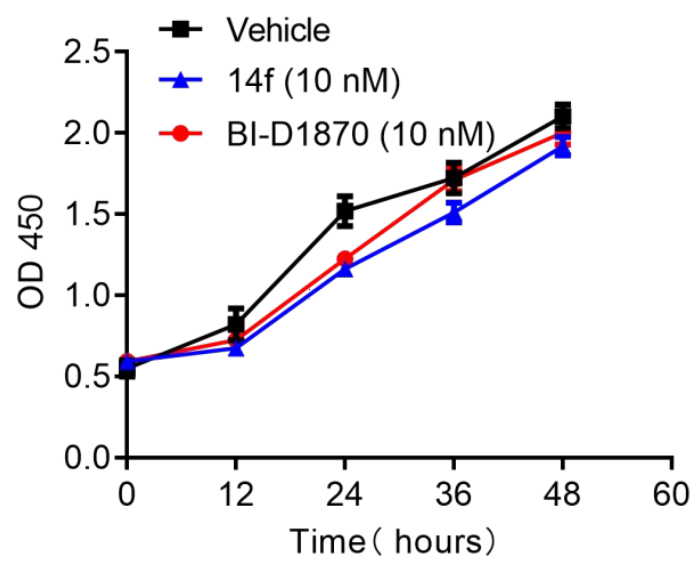

Figure S3. In vitro antiproliferation assay at $10 \mathrm{nM}$ for $14 \mathrm{f}$. $14 \mathrm{f}$ exerted no difference on the antiproliferative effect of ESCC at the concentration of $10 \mathrm{nM}$ compared with the same concentration of BI-D1870. 


\begin{tabular}{|c|c|c|c|c|c|c|c|c|c|c|c|c|c|c|c|c|c|c|c|c|c|c|c|}
\hline \multicolumn{6}{|c|}{ Vehicle } & \multicolumn{6}{|c|}{$14 \mathrm{f}(25 \mathrm{mg} / \mathrm{kg})$} & \multicolumn{6}{|c|}{$14 f(50 \mathrm{mg} / \mathrm{kg})$} & & \multicolumn{4}{|c|}{ BI-D1870 (50mg/kg) } & \\
\hline 1 & 2 & 3 & 4 & 5 & 6 & 1 & 2 & 3 & 4 & 5 & & 1 & 2 & 3 & 4 & 5 & & & & & & & \\
\hline
\end{tabular}
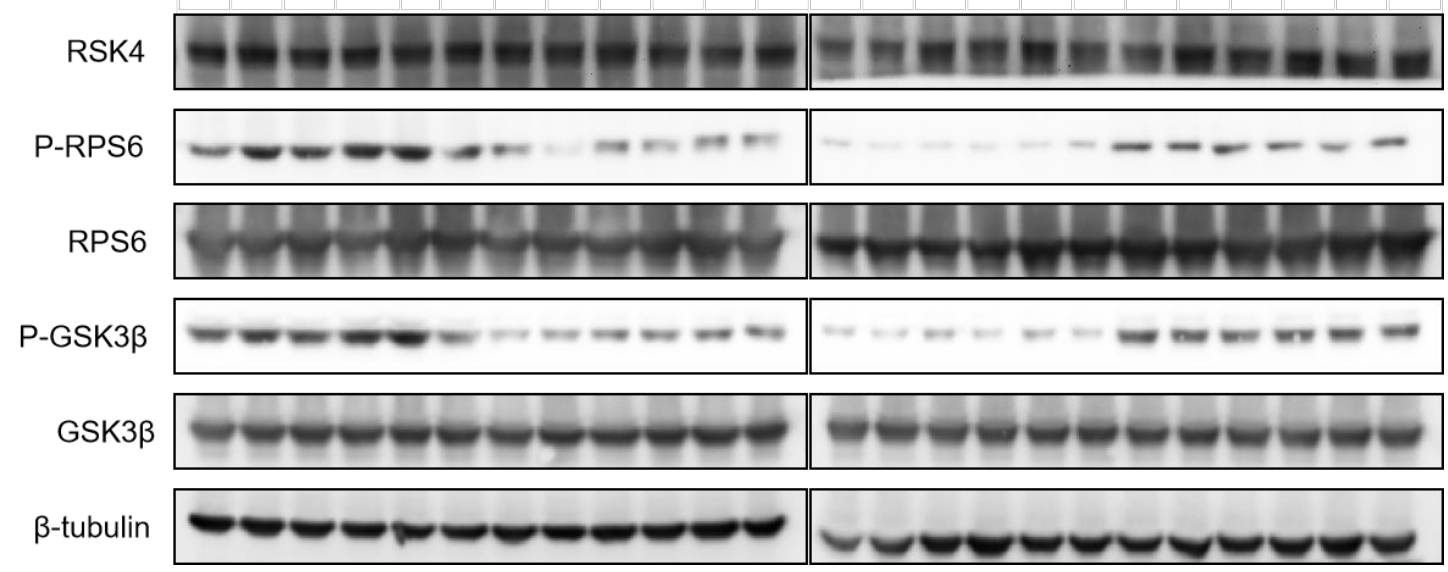

Figure S4. 14f inhibited the phosphorylation of RPS6 and GSK3 $\beta$ in tumor samples from the xenograft mouse models. 
Table S1. Kinase profiling results for $14 f$ at $100 \mathrm{nM}$.

\begin{tabular}{|c|c|c|}
\hline NO. & Gene Symbol & $\%$ Ctrl@100 nM \\
\hline 1 & AAK1 & 99 \\
\hline 2 & ABL1(E255K)-phosphorylated & 82 \\
\hline 3 & ABL1(F317I)-nonphosphorylated & 93 \\
\hline 4 & ABL1(F317I)-phosphorylated & 91 \\
\hline 5 & ABL1(F317L)-nonphosphorylated & 62 \\
\hline 6 & ABL1(F317L)-phosphorylated & 84 \\
\hline 7 & ABL1(H396P)-nonphosphorylated & 74 \\
\hline 8 & ABL1(H396P)-phosphorylated & 100 \\
\hline 9 & ABL1(M351T)-phosphorylated & 78 \\
\hline 10 & ABL1(Q252H)-nonphosphorylated & 73 \\
\hline 11 & ABL1(Q252H)-phosphorylated & 100 \\
\hline 12 & ABL1(T315I)-nonphosphorylated & 76 \\
\hline 13 & ABL1(T315I)-phosphorylated & 74 \\
\hline 14 & ABL1(Y253F)-phosphorylated & 77 \\
\hline 15 & ABL1-nonphosphorylated & 73 \\
\hline 16 & ABL1-phosphorylated & 90 \\
\hline 17 & ABL2 & 100 \\
\hline 18 & ACVR1 & 100 \\
\hline 19 & ACVR1B & 100 \\
\hline 20 & ACVR2A & 100 \\
\hline 21 & ACVR2B & 100 \\
\hline 22 & ACVRL1 & 65 \\
\hline 23 & ADCK3 & 100 \\
\hline 24 & ADCK4 & 100 \\
\hline 25 & AKT1 & 100 \\
\hline 26 & AKT2 & 100 \\
\hline 27 & AKT3 & 100 \\
\hline 28 & ALK & 94 \\
\hline 29 & ALK(C1156Y) & 82 \\
\hline 30 & ALK(L1196M) & 94 \\
\hline 31 & AMPK-alpha1 & 100 \\
\hline 32 & AMPK-alpha2 & 86 \\
\hline 33 & ANKK1 & 98 \\
\hline 34 & ARK5 & 100 \\
\hline 35 & ASK1 & 100 \\
\hline 36 & ASK2 & 89 \\
\hline 37 & AURKA & 94 \\
\hline 38 & AURKB & 85 \\
\hline 39 & AURKC & 100 \\
\hline 40 & AXL & 100 \\
\hline
\end{tabular}


41 BIKE 100

42 BLK 100

43 BMPR1A 94

44 BMPR1B $\quad 87$

45 BMPR2 83

46 BMX 100

47 BRAF 90

48 BRAF(V600E) 92

49 BRK 100

50 BRSK1 100

51 BRSK2 87

52 BTK 98

53 BUB1 100

54 CAMK1 100

55 CAMK1B 74

56 CAMK1D 100

57 CAMK1G 100

58 CAMK2A 100

59 CAMK2B 100

60 CAMK2D 100

61 CAMK2G 100

62 CAMK4 100

63 CAMKK1 100

64 CAMKK2 100

65 CASK 77

66 CDC2L1 100

67 CDC2L2 99

68 CDC2L5 88

69 CDK11 100

70 CDK2 100

$\begin{array}{lll}71 & \mathrm{CDK} 3 & 100\end{array}$

$\begin{array}{lll}72 & \text { CDK4 } & 100\end{array}$

73 CDK4-cyclinD1 100

74 CDK4-cyclinD3 98

75 CDK5 100

$76 \quad$ CDK7 67

77 CDK8 88

78 CDK9 100

79 CDKL1 76

$80 \quad$ CDKL2 93

81 CDKL3 100

82 CDKL5 97

83 CHEK1 100

84 CHEK2 50 


\begin{tabular}{|c|c|c|}
\hline 85 & CIT & 94 \\
\hline 86 & CLK1 & 100 \\
\hline 87 & CLK2 & 92 \\
\hline 88 & CLK3 & 100 \\
\hline 89 & CLK4 & 100 \\
\hline 90 & CSF1R & 98 \\
\hline 91 & CSF1R-autoinhibited & 66 \\
\hline 92 & CSK & 99 \\
\hline 93 & CSNK1A1 & 64 \\
\hline 94 & CSNK1A1L & 39 \\
\hline 95 & CSNK1D & 99 \\
\hline 96 & CSNK1E & 39 \\
\hline 97 & CSNK1G1 & 100 \\
\hline 98 & CSNK1G2 & 100 \\
\hline 99 & CSNK1G3 & 100 \\
\hline 100 & CSNK2A1 & 78 \\
\hline 101 & CSNK2A2 & 100 \\
\hline 102 & CTK & 80 \\
\hline 103 & DAPK 1 & 93 \\
\hline 104 & DAPK2 & 100 \\
\hline 105 & DAPK3 & 100 \\
\hline 106 & DCAMKL1 & 70 \\
\hline 107 & DCAMKL2 & 100 \\
\hline 108 & DCAMKL3 & 100 \\
\hline 109 & DDR1 & 99 \\
\hline 110 & DDR2 & 85 \\
\hline 111 & DLK & 78 \\
\hline 112 & DMPK & 100 \\
\hline 113 & DMPK2 & 100 \\
\hline 114 & DRAK1 & 100 \\
\hline 115 & DRAK2 & 100 \\
\hline 116 & DYRK1A & 88 \\
\hline 117 & DYRK1B & 72 \\
\hline 118 & DYRK2 & 80 \\
\hline 119 & EGFR & 100 \\
\hline 120 & EGFR(E746-A750del) & 85 \\
\hline 121 & EGFR(G719C) & 93 \\
\hline 122 & EGFR(G719S) & 87 \\
\hline 123 & EGFR(L747-E749del, A750P) & 100 \\
\hline 124 & EGFR(L747-S752del, P753S) & 90 \\
\hline 125 & EGFR(L747-T751del,Sins) & 94 \\
\hline 126 & EGFR(L858R) & 92 \\
\hline 127 & EGFR(L858R,T790M) & 92 \\
\hline 128 & EGFR(L861Q) & 97 \\
\hline
\end{tabular}


129 EGFR(S752-I759del) 89

130 EGFR(T790M) 96

131 EIF2AK1 100

132 EPHA1 100

133 EPHA2 100

134 EPHA3 100

135 EPHA4 95

136 EPHA5 100

137 EPHA6 98

138 EPHA7 100

139 EPHA8 100

140 EPHB1 100

141 EPHB2 100

142 EPHB3 100

143 EPHB4 99

144 EPHB6 95

145 ERBB2 86

146 ERBB3 91

147 ERBB4 93

148 ERK1 100

149 ERK2 100

150 ERK3 100

151 ERK4 100

152 ERK5 100

153 ERK8 100

154 ERN1 66

155 FAK 100

156 FER 96

157 FES 98

158 FGFR1 95

159 FGFR2 98

160 FGFR3 99

161 FGFR3(G697C) 100

162 FGFR4 100

163 FGR 100

164 FLT1 100

165 FLT3 100

166 FLT3(D835H) 69

167 FLT3(D835V) 54

168 FLT3(D835Y) 90

169 FLT3(ITD) 100

170 FLT3(ITD,D835V) 100

171 FLT3(ITD,F691L) 62

172 FLT3(K663Q) 93 


\begin{tabular}{|c|c|c|}
\hline 173 & FLT3(N841I) & 96 \\
\hline 174 & FLT3(R834Q) & 100 \\
\hline 175 & FLT3-autoinhibited & 86 \\
\hline 176 & FLT4 & 100 \\
\hline 177 & FRK & 100 \\
\hline 178 & FYN & 100 \\
\hline 179 & GAK & 85 \\
\hline 180 & GCN2(Kin.Dom.2,S808G) & 71 \\
\hline 181 & GRK1 & 86 \\
\hline 182 & GRK2 & 92 \\
\hline 183 & GRK3 & 88 \\
\hline 184 & GRK4 & 100 \\
\hline 185 & GRK7 & 98 \\
\hline 186 & GSK3A & 44 \\
\hline 187 & GSK3B & 65 \\
\hline 188 & HASPIN & 79 \\
\hline 189 & $\mathrm{HCK}$ & 100 \\
\hline 190 & HIPK1 & 90 \\
\hline 191 & HIPK2 & 94 \\
\hline 192 & HIPK3 & 93 \\
\hline 193 & HIPK4 & 100 \\
\hline 194 & HPK1 & 97 \\
\hline 195 & HUNK & 81 \\
\hline 196 & ICK & 80 \\
\hline 197 & IGF1R & 100 \\
\hline 198 & IKK-alpha & 94 \\
\hline 199 & IKK-beta & 86 \\
\hline 200 & IKK-epsilon & 75 \\
\hline 201 & INSR & 100 \\
\hline 202 & INSRR & 100 \\
\hline 203 & IRAK1 & 87 \\
\hline 204 & IRAK3 & 100 \\
\hline 205 & IRAK4 & 91 \\
\hline 206 & ITK & 100 \\
\hline 207 & JAK1(JH1domain-catalytic) & 100 \\
\hline 208 & JAK1(JH2domain-pseudokinase) & 95 \\
\hline 209 & JAK2(JH1domain-catalytic) & 100 \\
\hline 210 & JAK3(JH1domain-catalytic) & 97 \\
\hline 211 & JNK1 & 78 \\
\hline 212 & JNK2 & 88 \\
\hline 213 & JNK3 & 74 \\
\hline 214 & KIT & 100 \\
\hline 215 & KIT(A829P) & 83 \\
\hline 216 & KIT(D816H) & 87 \\
\hline
\end{tabular}




\begin{tabular}{|c|c|c|}
\hline 217 & KIT(D816V) & 100 \\
\hline 218 & KIT(L576P) & 100 \\
\hline 219 & KIT(V559D) & 100 \\
\hline 220 & KIT(V559D,T670I) & 100 \\
\hline 221 & KIT(V559D,V654A) & 98 \\
\hline 222 & KIT-autoinhibited & 83 \\
\hline 223 & LATS1 & 100 \\
\hline 224 & LATS2 & 81 \\
\hline 225 & LCK & 100 \\
\hline 226 & LIMK1 & 94 \\
\hline 227 & LIMK2 & 100 \\
\hline 228 & LKB1 & 52 \\
\hline 229 & LOK & 100 \\
\hline 230 & LRRK2 & 85 \\
\hline 231 & LRRK2(G2019S) & 72 \\
\hline 232 & LTK & 80 \\
\hline 233 & LYN & 100 \\
\hline 234 & LZK & 91 \\
\hline 235 & MAK & 100 \\
\hline 236 & MAP3K1 & 94 \\
\hline 237 & MAP3K15 & 86 \\
\hline 238 & MAP3K2 & 89 \\
\hline 239 & MAP3K3 & 84 \\
\hline 240 & MAP3K4 & 100 \\
\hline 241 & MAP4K2 & 100 \\
\hline 242 & MAP4K3 & 97 \\
\hline 243 & MAP4K4 & 92 \\
\hline 244 & MAP4K5 & 100 \\
\hline 245 & MAPKAPK2 & 96 \\
\hline 246 & MAPKAPK5 & 87 \\
\hline 247 & MARK1 & 85 \\
\hline 248 & MARK2 & 100 \\
\hline 249 & MARK3 & 89 \\
\hline 250 & MARK4 & 94 \\
\hline 251 & MAST1 & 66 \\
\hline 252 & MEK1 & 97 \\
\hline 253 & MEK2 & 90 \\
\hline 254 & MEK3 & 71 \\
\hline 255 & MEK4 & 67 \\
\hline 256 & MEK5 & 79 \\
\hline 257 & MEK6 & 86 \\
\hline 258 & MELK & 33 \\
\hline 259 & MERTK & 61 \\
\hline 260 & MET & 100 \\
\hline
\end{tabular}


261 MET(M1250T) 100

262 MET(Y1235D) 100

263 MINK 78

264 MKK7 96

265 MKNK1 91

266 MKNK2 93

267 MLCK 100

268 MLK1 100

269 MLK2 93

270 MLK3 80

271 MRCKA 100

272 MRCKB 100

273 MST1 98

274 MST1R 100

275 MST2 98

276 MST3 100

277 MST4 84

278 MTOR $\quad 70$

279 MUSK 100

280 MYLK 91

281 MYLK2 98

282 MYLK4 92

283 MYO3A 100

284 MYO3B 100

285 NDR1 100

286 NDR2 100

287 NEK1 83

288 NEK10 100

289 NEK11 98

290 NEK2 100

291 NEK3 76

292 NEK4 94

293 NEK5 52

294 NEK6 74

295 NEK7 60

296 NEK9 65

297 NIK 71

298 NIM1 83

299 NLK 100

300 OSR1 98

301 p38-alpha 100

302 p38-beta 100

303 p38-delta 100

304 p38-gamma 91 


\begin{tabular}{|c|c|c|}
\hline 305 & PAK1 & 98 \\
\hline 306 & PAK2 & 96 \\
\hline 307 & PAK3 & 100 \\
\hline 308 & PAK4 & 100 \\
\hline 309 & PAK6 & 100 \\
\hline 310 & PAK7 & 100 \\
\hline 311 & PCTK1 & 71 \\
\hline 312 & PCTK2 & 95 \\
\hline 313 & PCTK3 & 88 \\
\hline 314 & PDGFRA & 66 \\
\hline 315 & PDGFRB & 99 \\
\hline 316 & PDPK1 & 100 \\
\hline 317 & PFCDPK1(P.falciparum) & 72 \\
\hline 318 & PFPK5(P.falciparum) & 82 \\
\hline 319 & PFTAIRE2 & 97 \\
\hline 320 & PFTK1 & 95 \\
\hline 321 & PHKG1 & 100 \\
\hline 322 & PHKG2 & 100 \\
\hline 323 & PIK3C2B & 100 \\
\hline 324 & PIK3C2G & 71 \\
\hline 325 & PIK3CA & 100 \\
\hline 326 & PIK3CA(C420R) & 97 \\
\hline 327 & PIK3CA(E542K) & 78 \\
\hline 328 & PIK3CA(E545A) & 89 \\
\hline 329 & PIK3CA(E545K) & 83 \\
\hline 330 & PIK3CA(H1047L) & 80 \\
\hline 331 & PIK3CA(H1047Y) & 58 \\
\hline 332 & PIK3CA(I800L) & 90 \\
\hline 333 & PIK3CA(M1043I) & 87 \\
\hline 334 & PIK3CA(Q546K) & 91 \\
\hline 335 & PIK3CB & 100 \\
\hline 336 & PIK3CD & 75 \\
\hline 337 & PIK3CG & 99 \\
\hline 338 & PIK4CB & 63 \\
\hline 339 & PIKFYVE & 68 \\
\hline 340 & PIM1 & 100 \\
\hline 341 & PIM2 & 100 \\
\hline 342 & PIM3 & 92 \\
\hline 343 & PIP5K1A & 100 \\
\hline 344 & PIP5K1C & 63 \\
\hline 345 & PIP5K2B & 100 \\
\hline 346 & PIP5K2C & 19 \\
\hline 347 & PKAC-alpha & 100 \\
\hline 348 & PKAC-beta & 83 \\
\hline
\end{tabular}




\begin{tabular}{|c|c|c|}
\hline 349 & PKMYT1 & 100 \\
\hline 350 & PKN1 & 96 \\
\hline 351 & PKN2 & 99 \\
\hline 352 & PKNB(M.tuberculosis) & 100 \\
\hline 353 & PLK1 & 62 \\
\hline 354 & PLK2 & 83 \\
\hline 355 & PLK3 & 66 \\
\hline 356 & PLK4 & 100 \\
\hline 357 & PRKCD & 100 \\
\hline 358 & PRKCE & 91 \\
\hline 359 & PRKCH & 100 \\
\hline 360 & PRKCI & 84 \\
\hline 361 & PRKCQ & 100 \\
\hline 362 & PRKD1 & 0 \\
\hline 363 & PRKD2 & 100 \\
\hline 364 & PRKD3 & 95 \\
\hline 365 & PRKG1 & 96 \\
\hline 366 & PRKG2 & 97 \\
\hline 367 & PRKR & 86 \\
\hline 368 & PRKX & 100 \\
\hline 369 & PRP4 & 69 \\
\hline 370 & PYK2 & 100 \\
\hline 371 & QSK & 69 \\
\hline 372 & RAF1 & 100 \\
\hline 373 & RET & 100 \\
\hline 374 & RET(M918T) & 100 \\
\hline 375 & RET(V804L) & 98 \\
\hline 376 & RET(V804M) & 92 \\
\hline 377 & RIOK1 & 100 \\
\hline 378 & RIOK2 & 85 \\
\hline 379 & RIOK3 & 100 \\
\hline 380 & RIPK1 & 96 \\
\hline 381 & RIPK2 & 100 \\
\hline 382 & RIPK4 & 73 \\
\hline 383 & RIPK5 & 92 \\
\hline 384 & ROCK1 & 99 \\
\hline 385 & ROCK2 & 100 \\
\hline 386 & ROS1 & 92 \\
\hline 387 & RPS6KA4(Kin.Dom.1-N-terminal) & 31 \\
\hline 388 & RPS6KA4(Kin.Dom.2-C-terminal) & 99 \\
\hline 389 & RPS6KA5(Kin.Dom.1-N-terminal) & 100 \\
\hline 390 & RPS6KA5(Kin.Dom.2-C-terminal) & 92 \\
\hline 391 & RSK1(Kin.Dom.1-N-terminal) & 8.1 \\
\hline 392 & RSK1(Kin.Dom.2-C-terminal) & 100 \\
\hline
\end{tabular}




\begin{tabular}{|c|c|c|}
\hline 393 & RSK2(Kin.Dom.1-N-terminal) & 0.7 \\
\hline 394 & RSK2(Kin.Dom.2-C-terminal) & 100 \\
\hline 395 & RSK3(Kin.Dom.1-N-terminal) & 1.9 \\
\hline 396 & RSK3(Kin.Dom.2-C-terminal) & 100 \\
\hline 397 & RSK4(Kin.Dom.1-N-terminal) & 2.9 \\
\hline 398 & RSK4(Kin.Dom.2-C-terminal) & 99 \\
\hline 399 & S6K1 & 69 \\
\hline 400 & SBK1 & 82 \\
\hline 401 & SGK & 96 \\
\hline 402 & SgK110 & 99 \\
\hline 403 & SGK2 & 94 \\
\hline 404 & SGK3 & 90 \\
\hline 405 & SIK & 100 \\
\hline 406 & SIK2 & 98 \\
\hline 407 & SLK & 100 \\
\hline 408 & SNARK & 94 \\
\hline 409 & SNRK & 97 \\
\hline 410 & SRC & 100 \\
\hline 411 & SRMS & 90 \\
\hline 412 & SRPK1 & 99 \\
\hline 413 & SRPK2 & 98 \\
\hline 414 & SRPK3 & 100 \\
\hline 415 & STK16 & 100 \\
\hline 416 & STK33 & 100 \\
\hline 417 & STK35 & 98 \\
\hline 418 & STK36 & 100 \\
\hline 419 & STK39 & 100 \\
\hline 420 & SYK & 99 \\
\hline 421 & TAK1 & 87 \\
\hline 422 & TAOK1 & 62 \\
\hline 423 & TAOK2 & 81 \\
\hline 424 & TAOK3 & 99 \\
\hline 425 & TBK1 & 88 \\
\hline 426 & TEC & 100 \\
\hline 427 & TESK1 & 100 \\
\hline 428 & TGFBR1 & 100 \\
\hline 429 & TGFBR2 & 100 \\
\hline 430 & TIE1 & 100 \\
\hline 431 & TIE2 & 95 \\
\hline 432 & TLK1 & 92 \\
\hline 433 & TLK2 & 100 \\
\hline 434 & TNIK & 93 \\
\hline 435 & TNK1 & 98 \\
\hline 436 & TNK2 & 100 \\
\hline
\end{tabular}




\begin{tabular}{|c|c|c|}
\hline 437 & TNNI3K & 100 \\
\hline 438 & TRKA & 86 \\
\hline 439 & TRKB & 82 \\
\hline 440 & TRKC & 87 \\
\hline 441 & TRPM6 & 99 \\
\hline 442 & TSSK1B & 100 \\
\hline 443 & TSSK3 & 95 \\
\hline 444 & TTK & 42 \\
\hline 445 & TXK & 100 \\
\hline 446 & TYK2(JH1domain-catalytic) & 92 \\
\hline 447 & TYK2(JH2domain-pseudokinase) & 88 \\
\hline 448 & TYRO3 & 86 \\
\hline 449 & ULK1 & 83 \\
\hline 450 & ULK2 & 100 \\
\hline 451 & ULK3 & 91 \\
\hline 452 & VEGFR2 & 76 \\
\hline 453 & VPS34 & 96 \\
\hline 454 & VRK2 & 75 \\
\hline 455 & WEE1 & 97 \\
\hline 456 & WEE2 & 100 \\
\hline 457 & WNK1 & 100 \\
\hline 458 & WNK2 & 100 \\
\hline 459 & WNK3 & 100 \\
\hline 460 & WNK4 & 97 \\
\hline 461 & YANK1 & 98 \\
\hline 462 & YANK2 & 100 \\
\hline 463 & YANK3 & 100 \\
\hline 464 & YES & 98 \\
\hline 465 & YSK1 & 89 \\
\hline 466 & YSK4 & 69 \\
\hline 467 & ZAK & 100 \\
\hline 468 & ZAP70 & 77 \\
\hline
\end{tabular}


Table S2. Predicted lipophilicity and permeability properties of representative compounds ${ }^{a}$.

\begin{tabular}{|c|c|c|c|}
\hline Compounds & $\operatorname{logP}$ & QPlogPCaCo & QPPMDCK \\
\hline $\mathbf{1 4 a}$ & 3.86 & 743.58 & 1068.79 \\
\hline $\mathbf{1 4 f}$ & 3.60 & 878.75 & 1280.16 \\
\hline $\mathbf{1 4 g}$ & 3.71 & 548.47 & 587.38 \\
\hline $\mathbf{1 4 h}$ & 4.20 & 687.88 & 861.80 \\
\hline $\mathbf{1 4 s}$ & 5.85 & 2667.92 & 10000.00 \\
\hline
\end{tabular}

${ }^{a}$ The properties are predicted by QikProp module in Maestro (Schrödinger Inc, version 10.1). $\log \mathrm{P}$ equals to $\mathrm{QP} \log \mathrm{Po} / \mathrm{w}$ : Predicted octanol/water partition coefficient $(-2.0-$ 6.5); QPPCaco: Predicted apparent Caco- 2 cell permeability in $\mathrm{nm} / \mathrm{sec}(<25$ poor, $>500$ great); QPPMDCK: Predicted apparent MDCK cell permeability in nm/sec $(<25$ poor, $>500$ great). 
Table S3. Pharmacokinetics profiles of $14 \mathrm{f}$ in rats.

\begin{tabular}{|c|c|}
\hline Parameter $^{\mathbf{a}}$ & $\mathbf{1 4 f}$ \\
\hline IV dose $(\mathrm{mg} / \mathrm{kg})$ & 1 \\
\hline $\mathrm{C}_{0}(\mu \mathrm{g} / \mathrm{L})$ & $1513.18 \pm 290.66$ \\
\hline $\mathrm{T}_{1 / 2}(\mathrm{~h})$ & $0.92 \pm 0.09$ \\
\hline $\mathrm{AUC}_{0-\infty}\left(\mu \mathrm{g}^{*} \mathrm{~h} / \mathrm{L}\right)$ & $251.48 \pm 43.47$ \\
\hline $\mathrm{Cl}(\mathrm{L} / \mathrm{h} / \mathrm{kg})$ & $4.05 \pm 0.64$ \\
\hline $\mathrm{V}_{\mathrm{ss}}(\mathrm{L} / \mathrm{kg})$ & $1.28 \pm 0.27$ \\
\hline $\mathrm{PO} \mathrm{dose}(\mathrm{mg} / \mathrm{kg})$ & 10 \\
\hline $\mathrm{T}_{1 / 2}(\mathrm{~h})$ & $8.38 \pm \mathrm{NA}$ \\
\hline $\mathrm{T}_{\max }(\mathrm{h})$ & $3.42 \pm 4.06$ \\
\hline $\mathrm{C}_{\max }(\mu \mathrm{g} / \mathrm{L})$ & $3.82 \pm 2.99$ \\
\hline $\mathrm{AUC}_{0-\infty}\left(\mu \mathrm{g}^{*} \mathrm{~h} / \mathrm{L}\right)$ & $24.91 \pm \mathrm{NA}$ \\
\hline$F(\%)$ & 0.99 \\
\hline
\end{tabular}

a Compounds were dosed to an equal number of male Sprague-Dawley rats by intravenous (IV) and oral (PO) administration, respectively $(n=3)$. 
The spectra of ${ }^{1} \mathrm{H}$ NMR, ${ }^{13} \mathrm{C}$ NMR, HRMS (ESI) of all compounds and HPLC of representative compounds.

\section{Compound 14a}

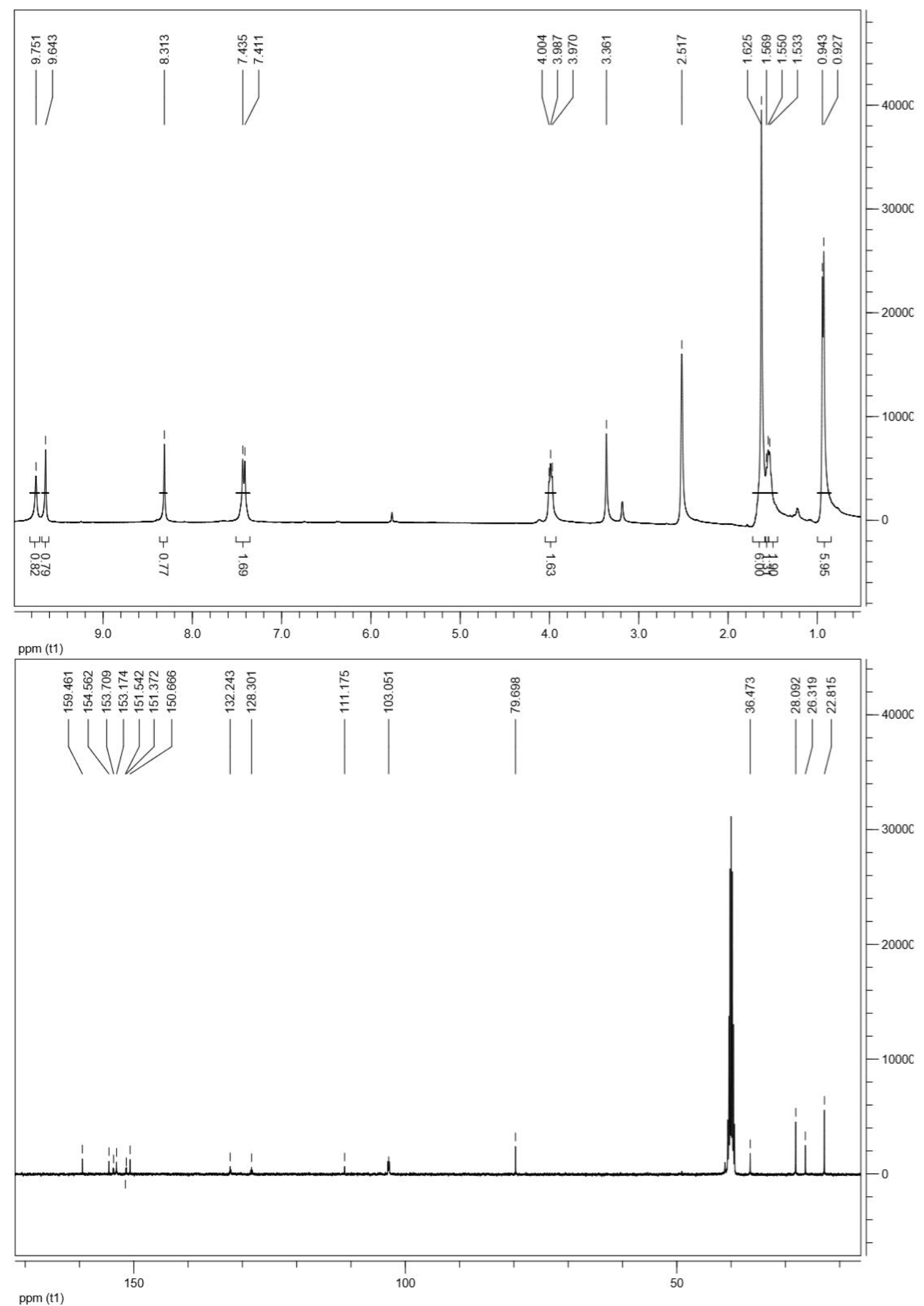




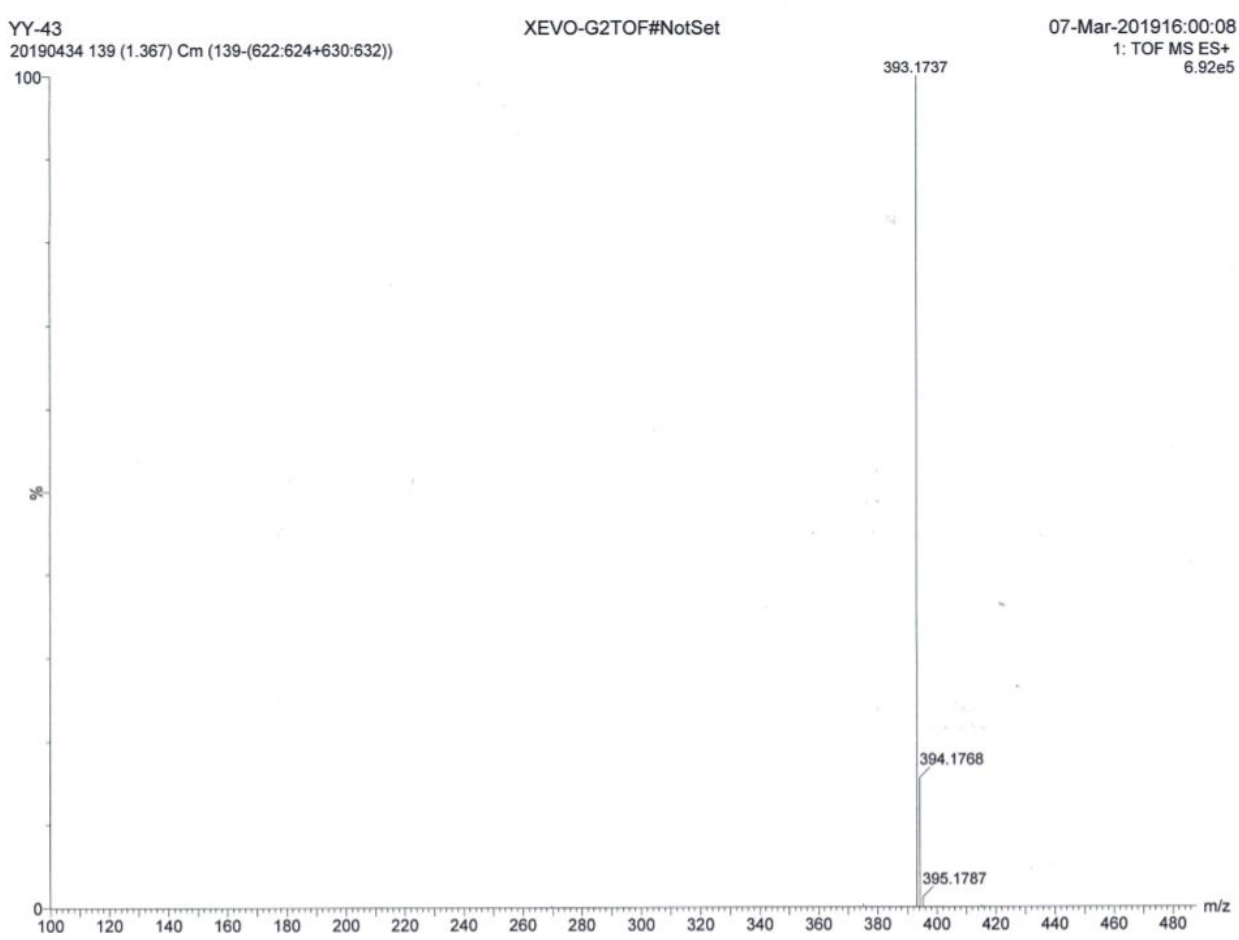

Acq.Operator $: Y Y$

Injection Date : Instrument $1 \quad$ Location: Sample 2

Instrument $\quad: 2020 / 1 / 14 \quad 16: 44: 50$

Method $\quad$ :D:ICHEM32\1 LMETHODSLLCX-DNA.M

Last changed $\quad: 2020 / 1 / 14 \quad 17: 08: 46:$ YY

Analyse $\quad$ : D:ICHEM32 1 IMETHODS LCX-DNA.M

Last changed $\quad: 2020 / 1 / 14 \quad 17: 11: 04: Y Y$

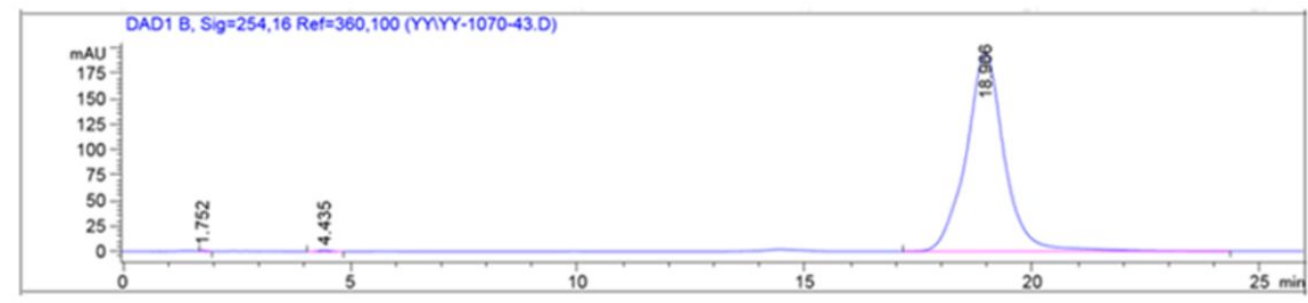

Sorted By $\quad$ : $\quad$ Signal

Multiplier $\quad: 1.0000$

Dilution $\quad: 1.0000$

Signal 2: DAD1 B, Sig=245,16 Ref $=360,100$

\begin{tabular}{|c|c|c|c|c|c|}
\hline $\begin{array}{c}\text { Peak } \\
\vdots\end{array}$ & $\begin{array}{l}\text { RetTime } \\
{[\min ]}\end{array}$ & Type Width & $\begin{array}{l}\text { Area } \\
{\left[m A U^{\star} s\right]}\end{array}$ & $\begin{array}{l}\text { Height } \\
\text { [mAU] }\end{array}$ & $\underset{8}{\text { Area }}$ \\
\hline 1 & 752 & 0.0854 & & 1.26870 & 0.0663 \\
\hline & 4.435 & & & & \\
\hline & 18.966 & 0.8489 & $1.13575 \mathrm{e} 4$ & 194.04416 & 99.7634 \\
\hline
\end{tabular}

Totals:

$1.13844 \mathrm{e} 4 \quad 196.61735$ 


\section{Compound 14b}

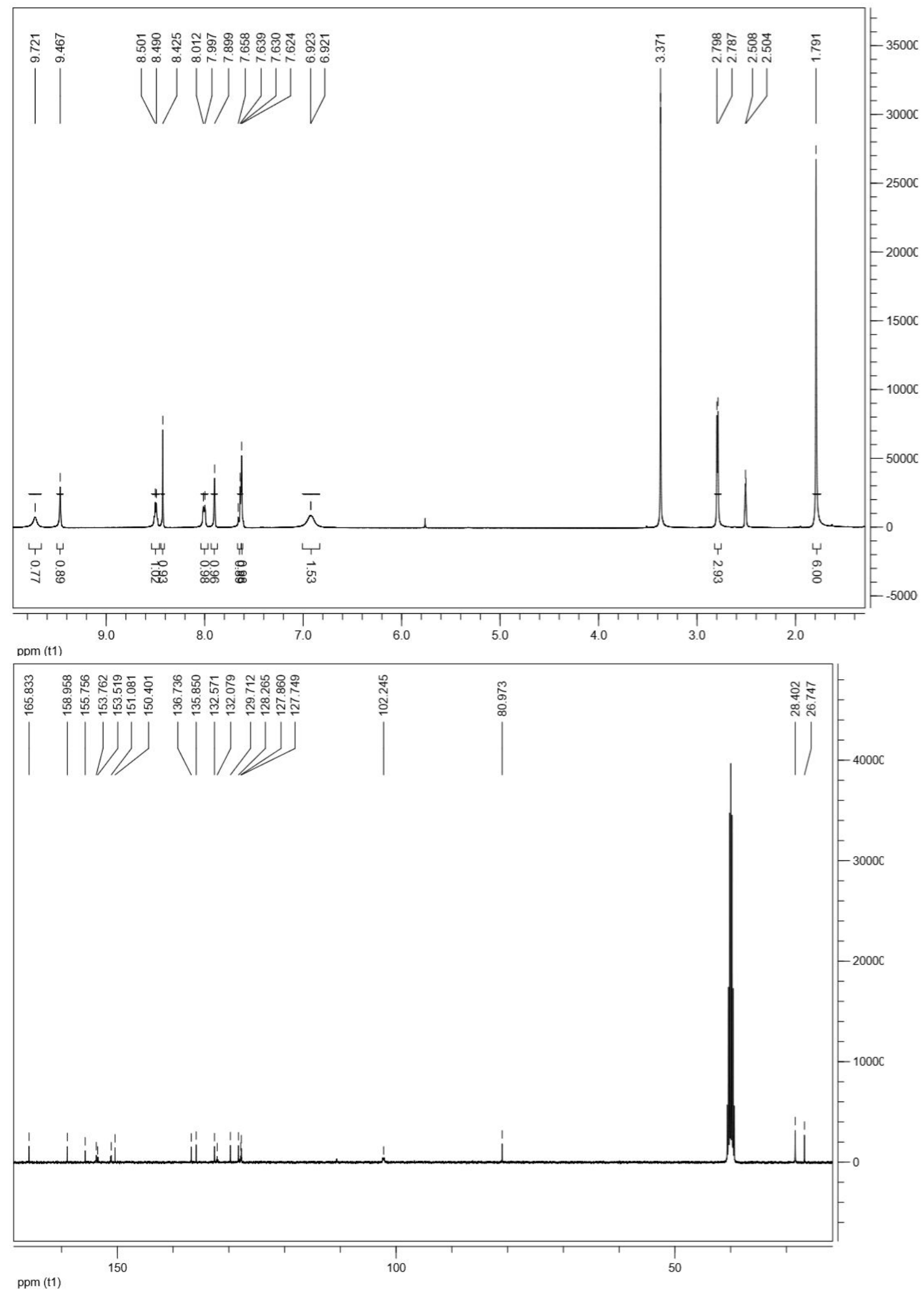




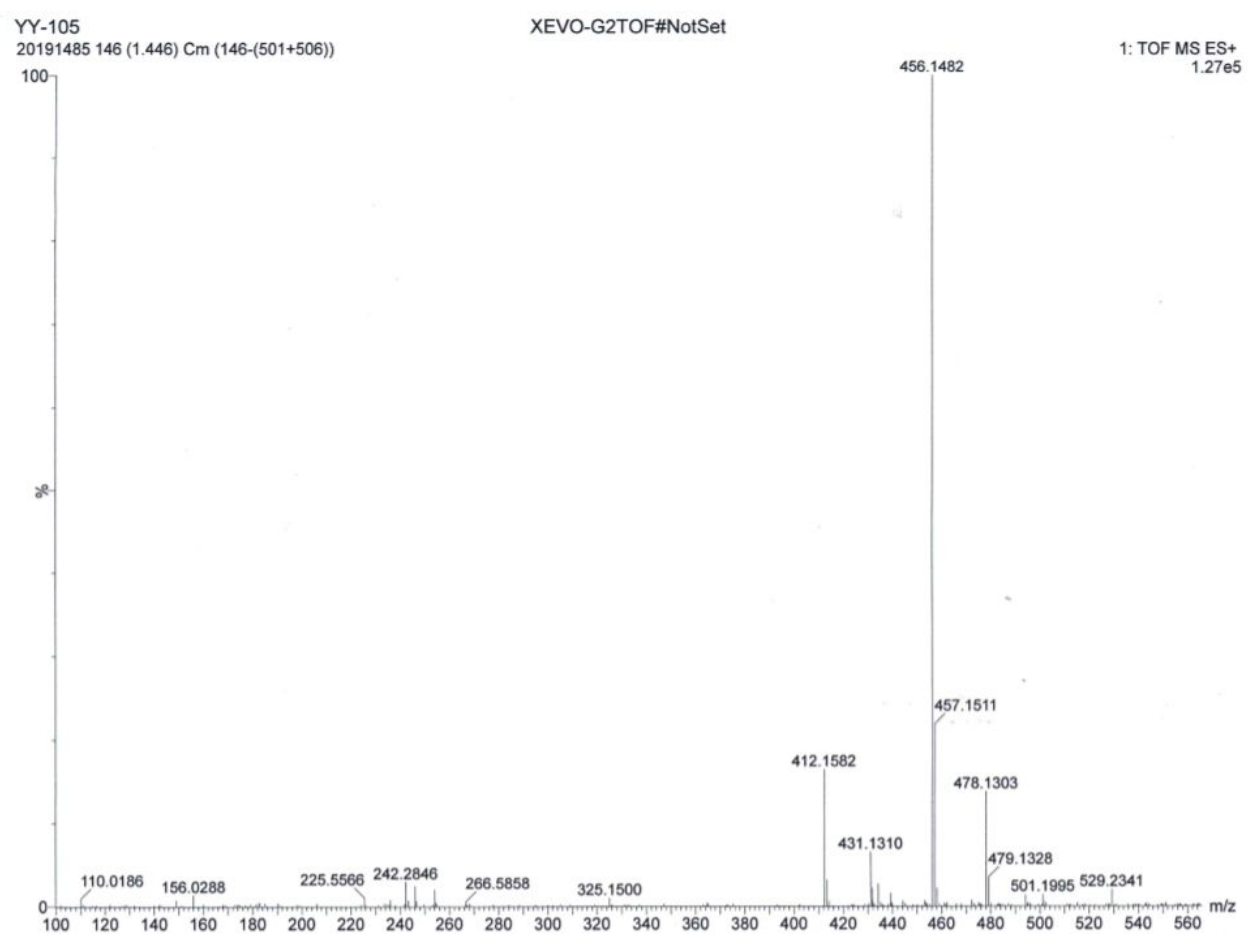

S23 


\section{Compound 14c}
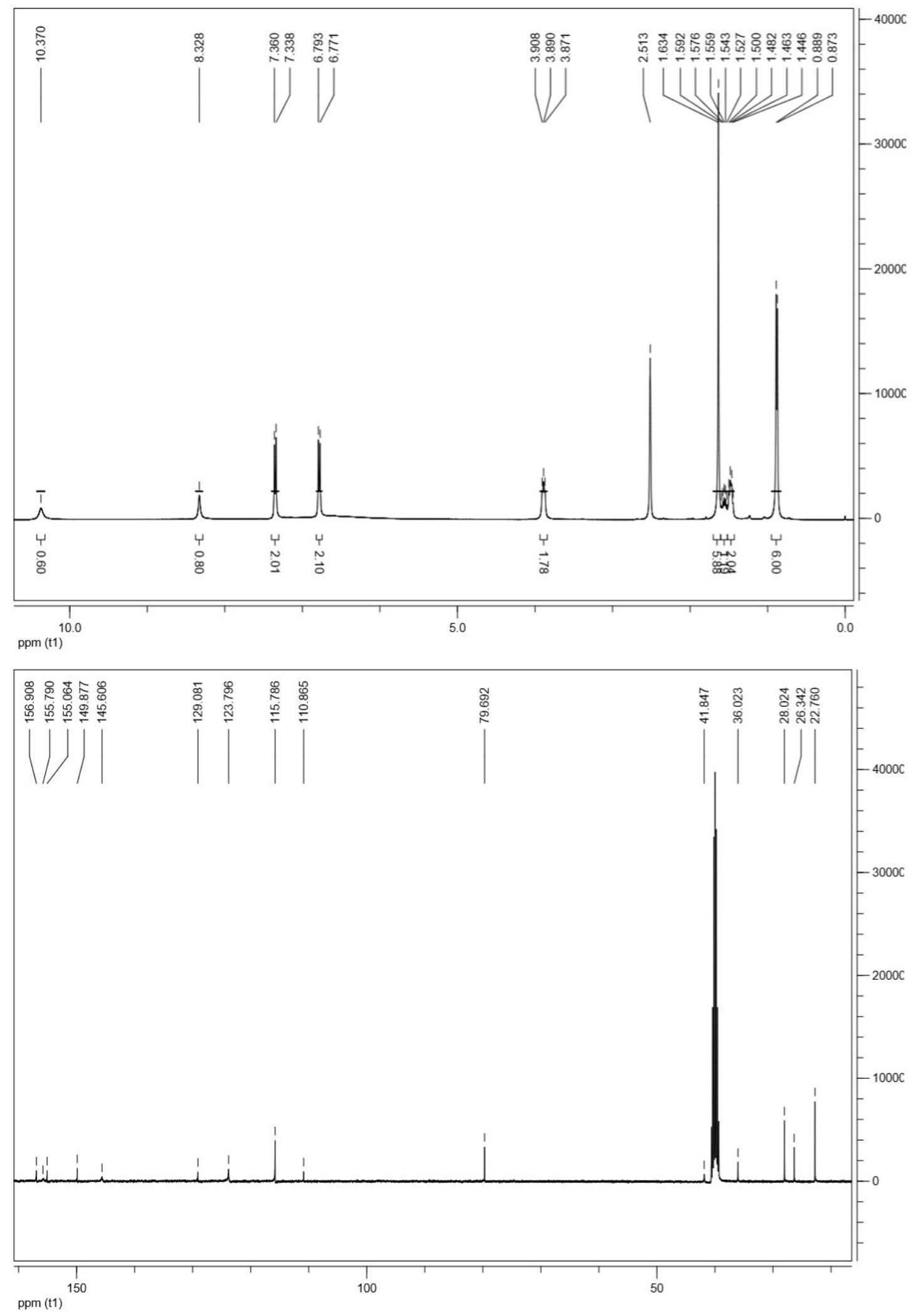


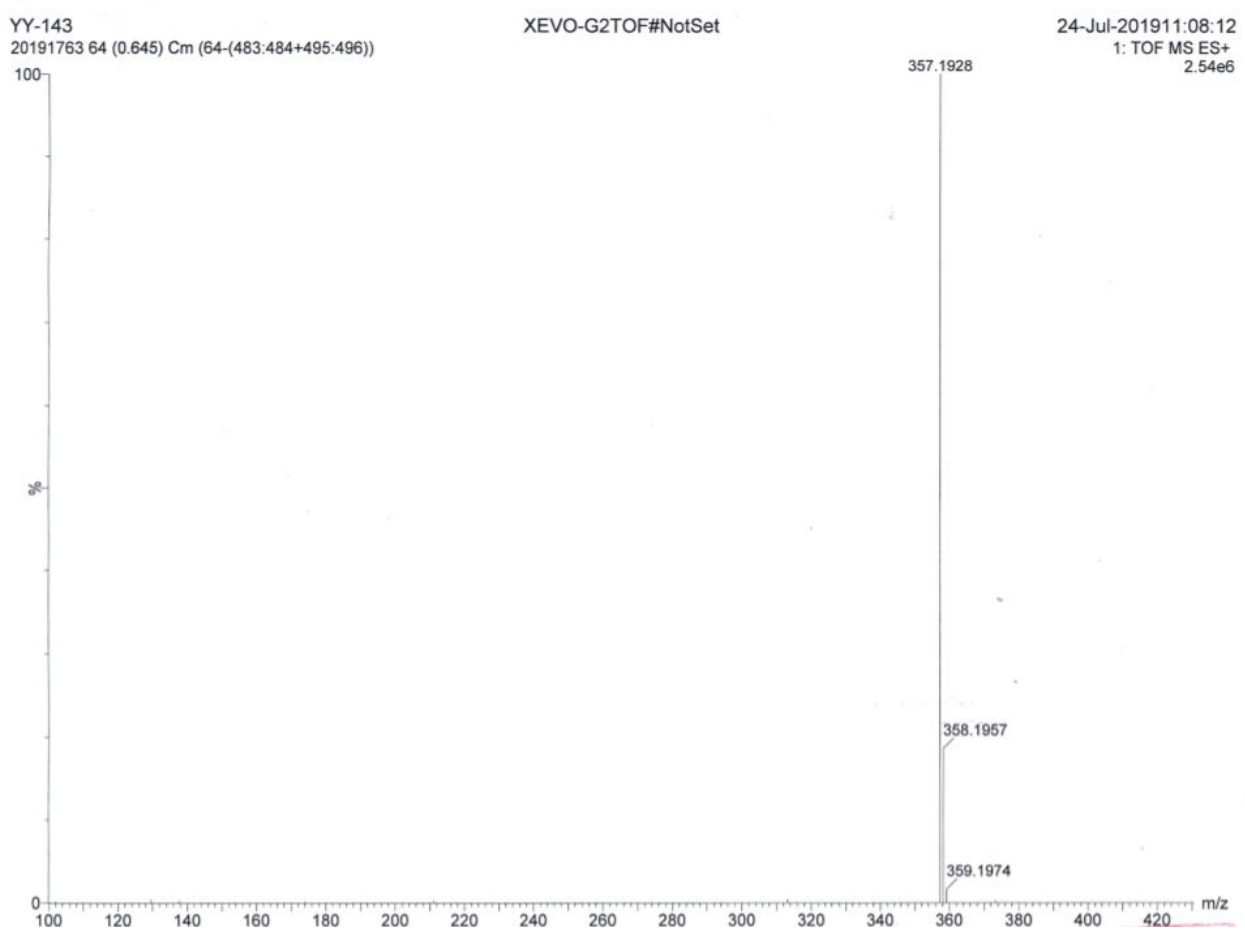




\section{Compound 14d}
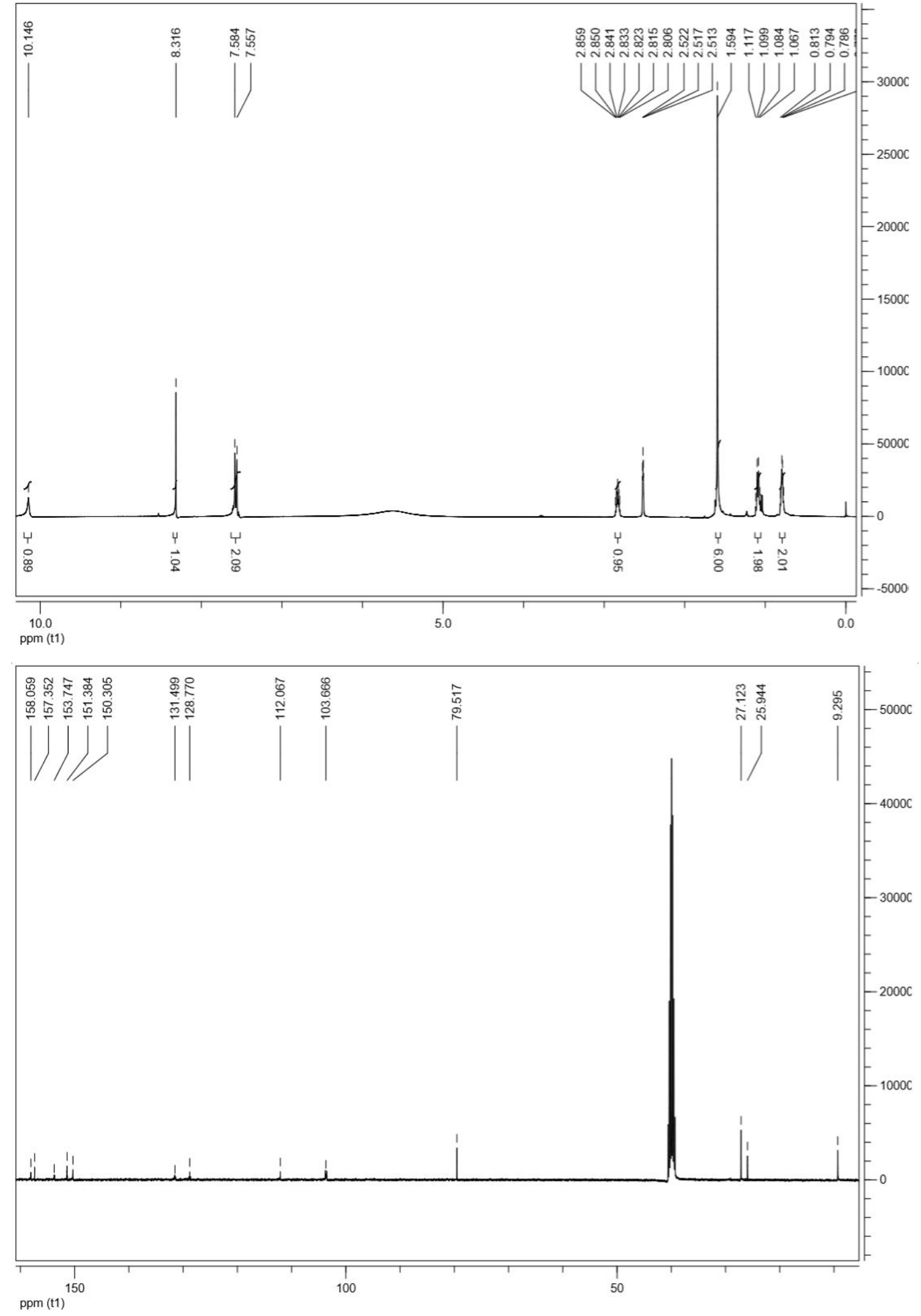
YY-1181-79

2020070720 (0.228) Cm (20-(477:479+485:487))

100

XEVO-G2TOF\#NotSet

363.1270

1: TOF MS ES+
$5.73 \mathrm{e} 5$

28-Jun-202013:50:44

$$
\text { 1: TOF MS ES+ }
$$

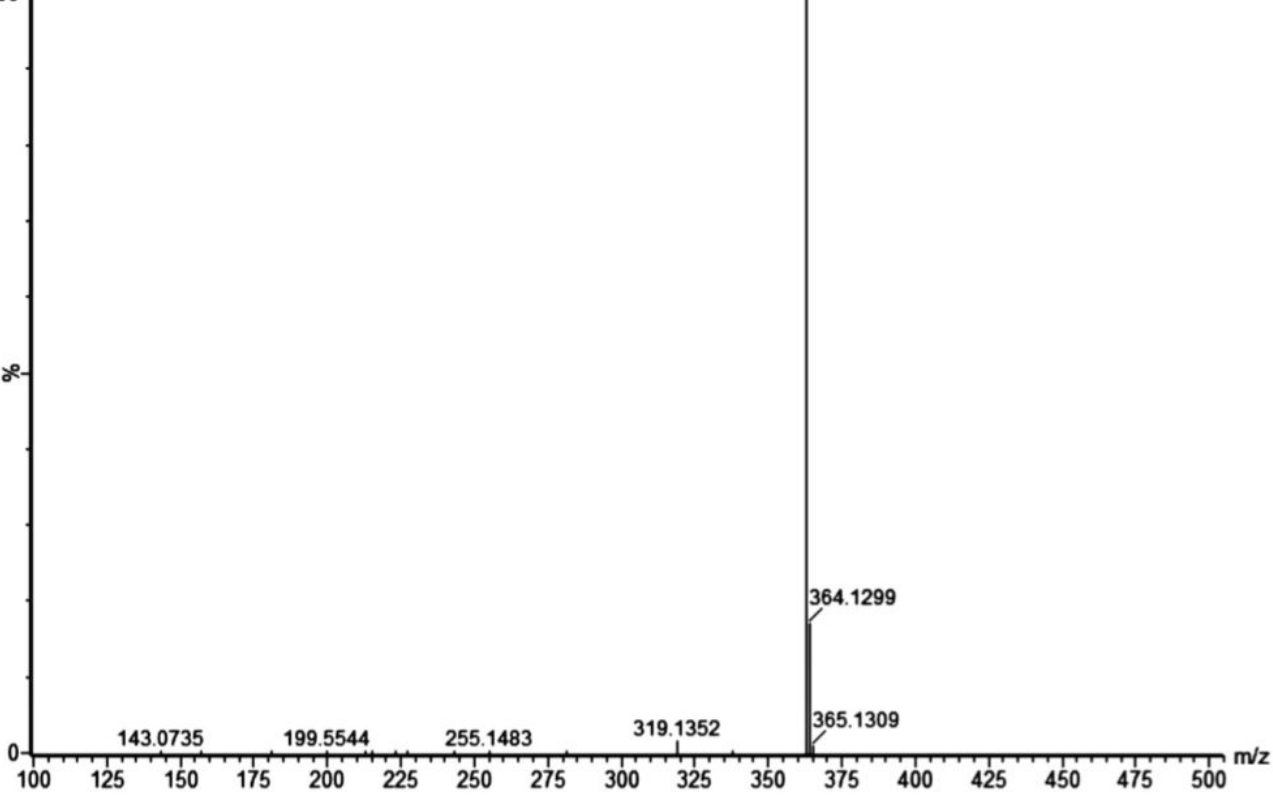




\section{Compound 14e}

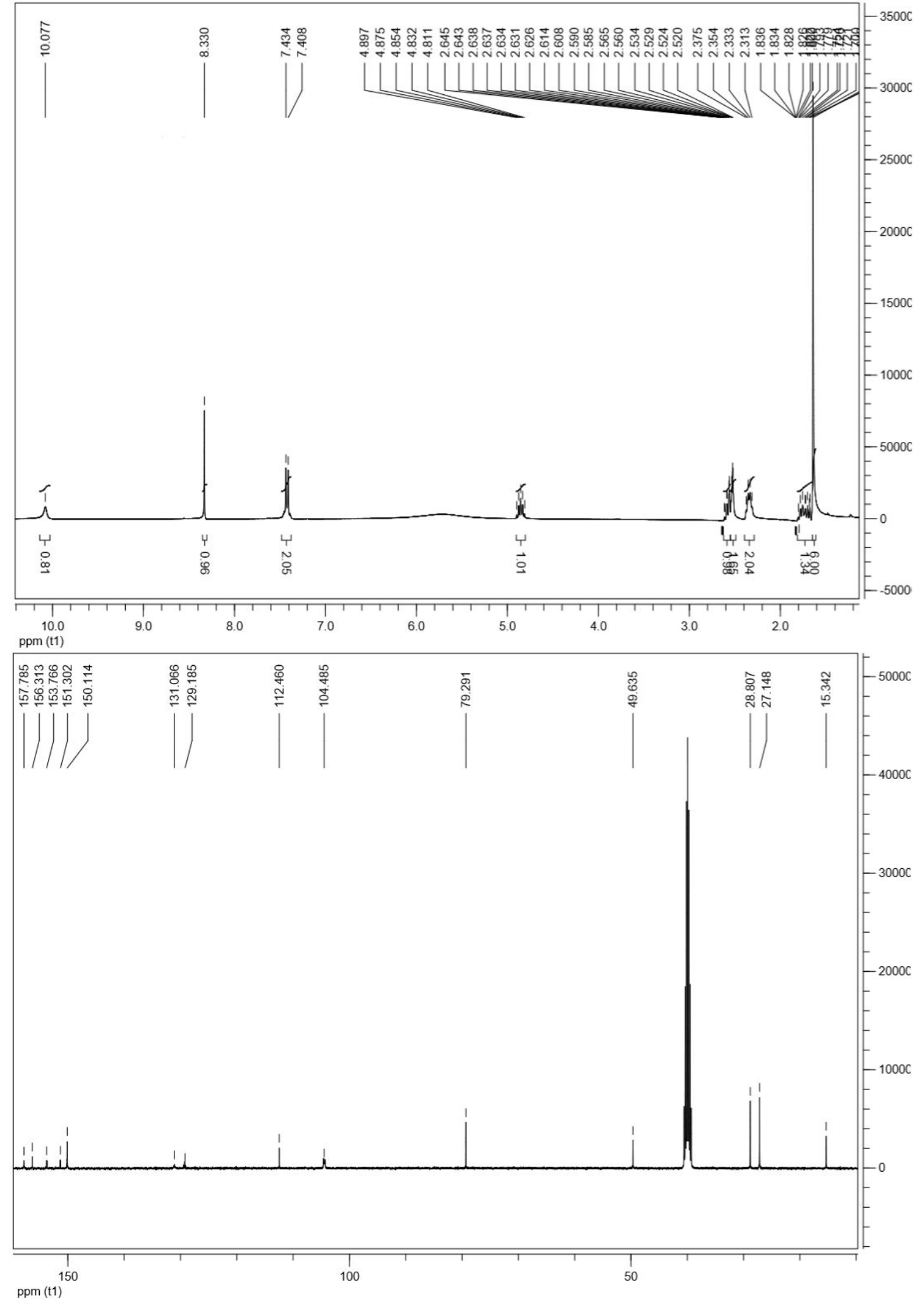




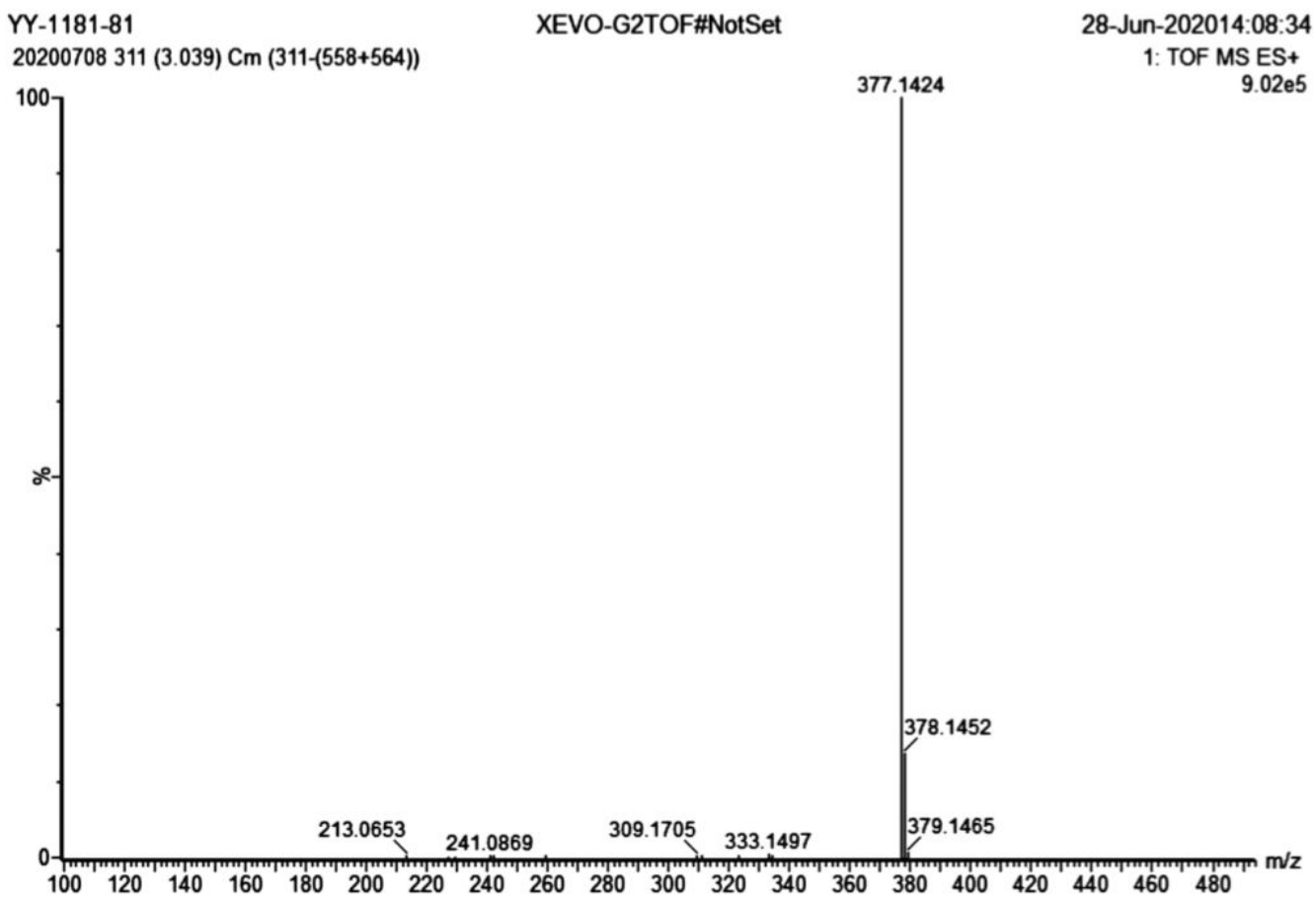




\section{Compound 14f}

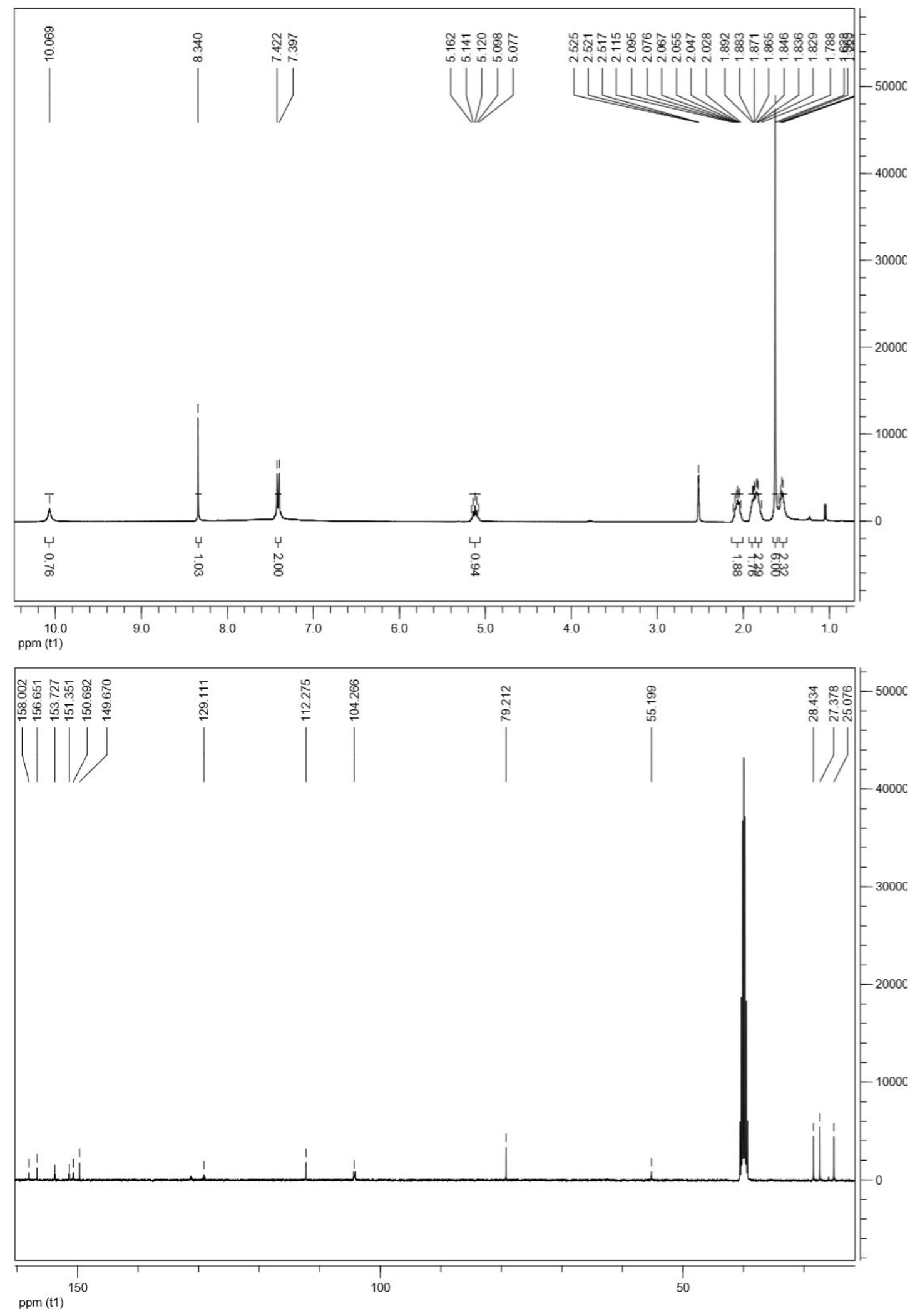



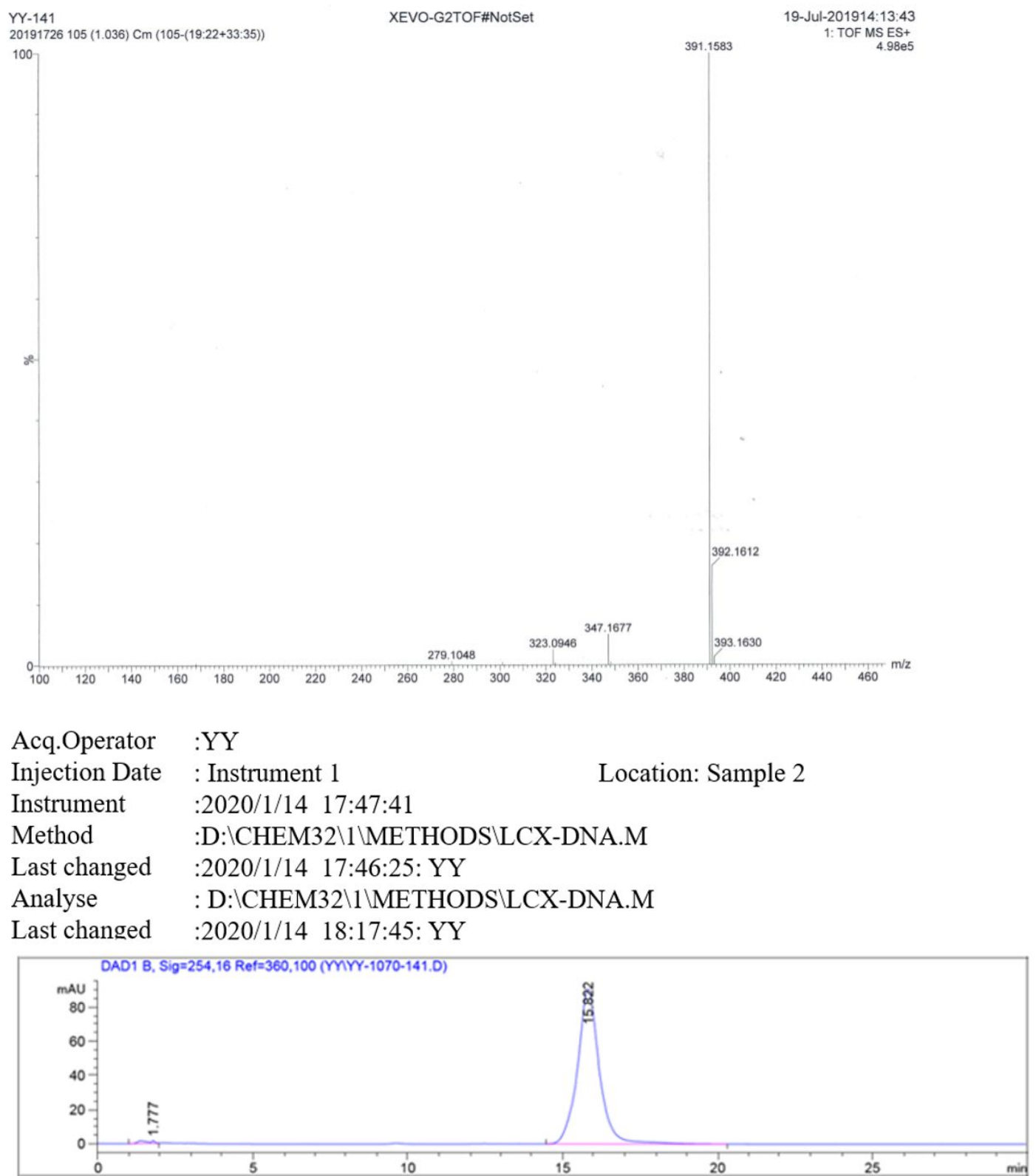

Sorted By $\quad: \quad$ Signal

Multiplier $\quad: 1.0000$

Dilution $\quad: 1.0000$

Signal 3: DAD1 B, Sig $=245,16$ Ref $=360,100$

Peak RetTime Type Width Area Height Area

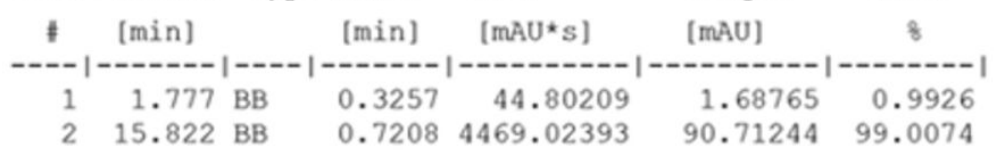

Totals:

$4513.82602 \quad 92.40009$ 
Compound 14g
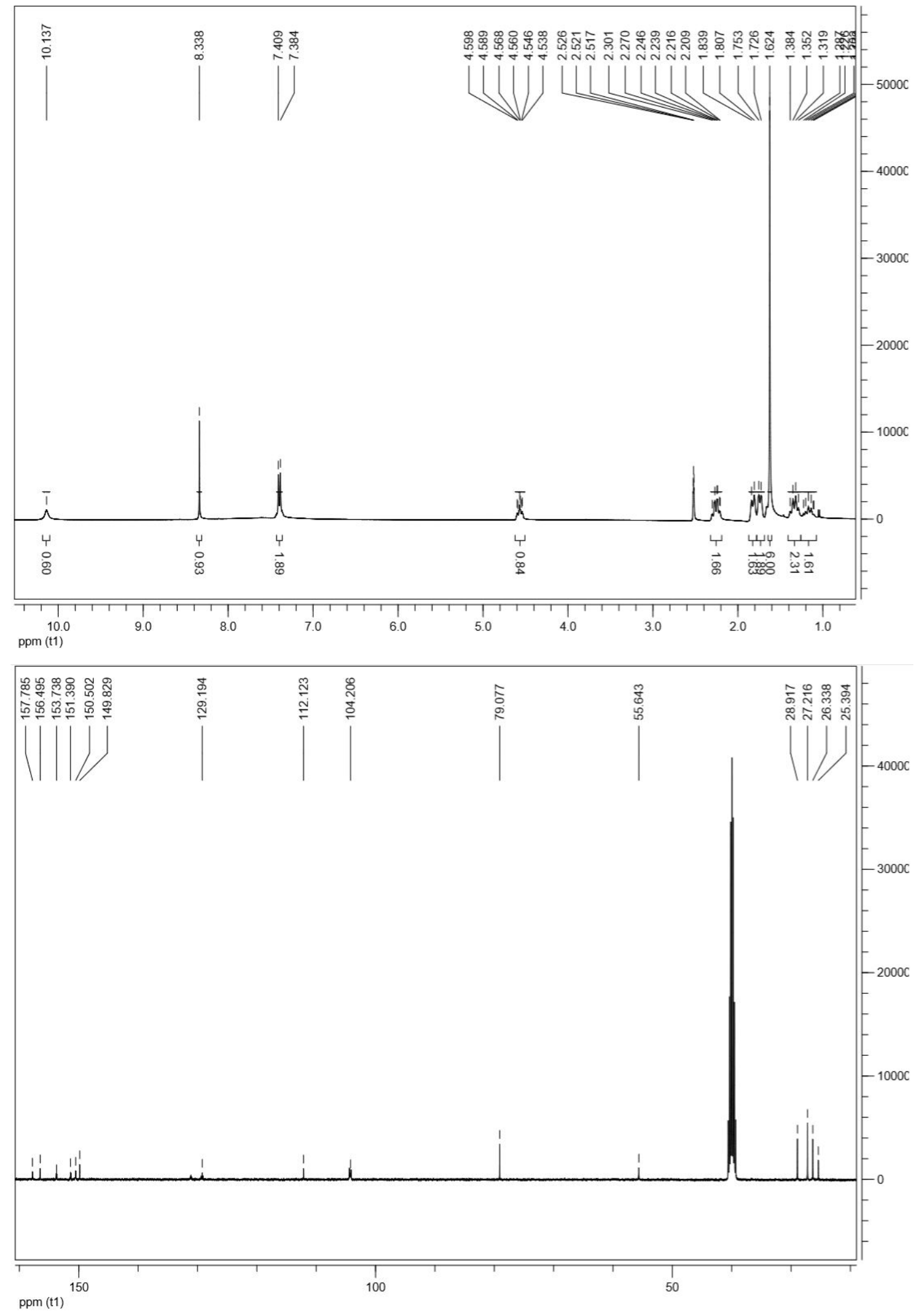


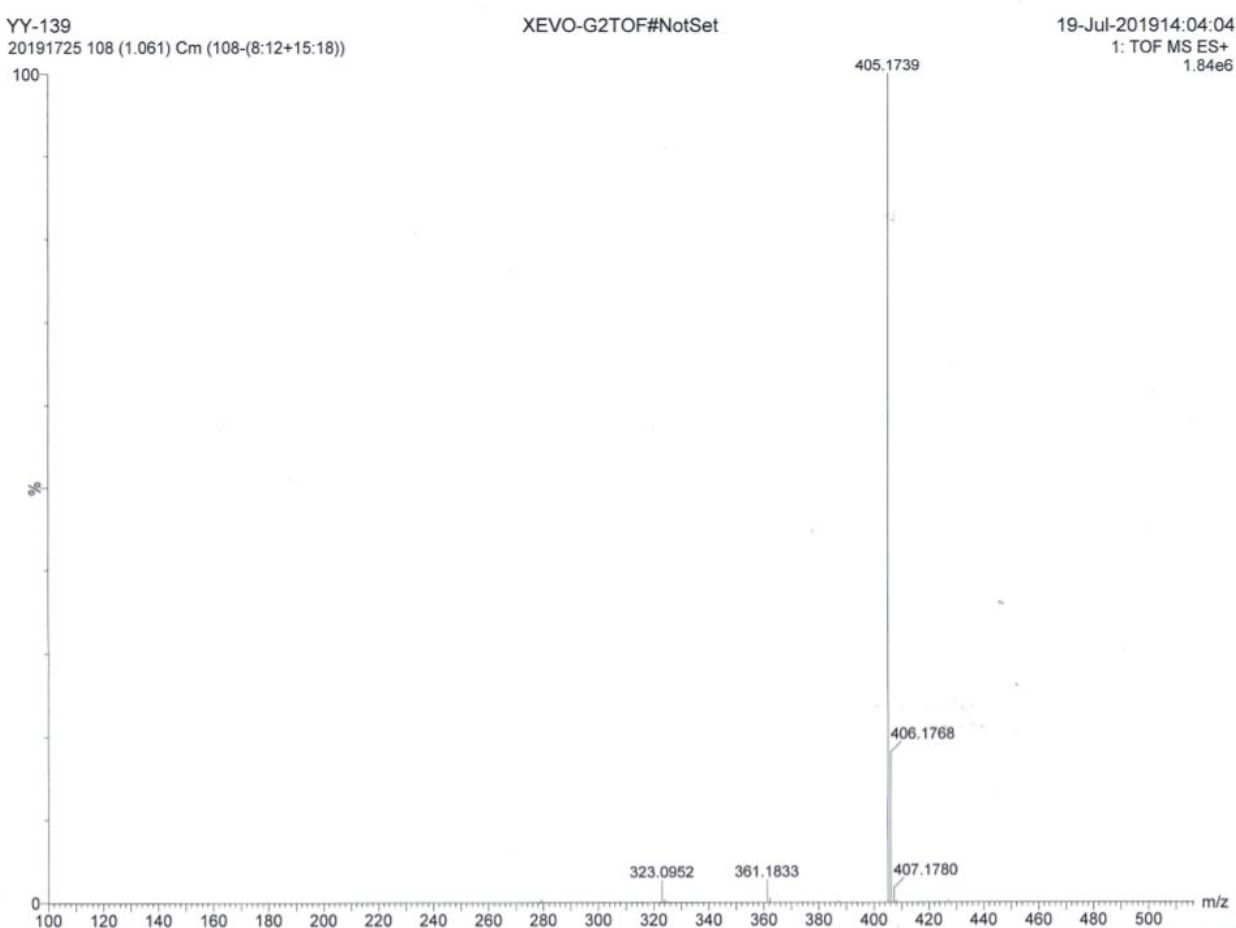




\section{Compound 14h}

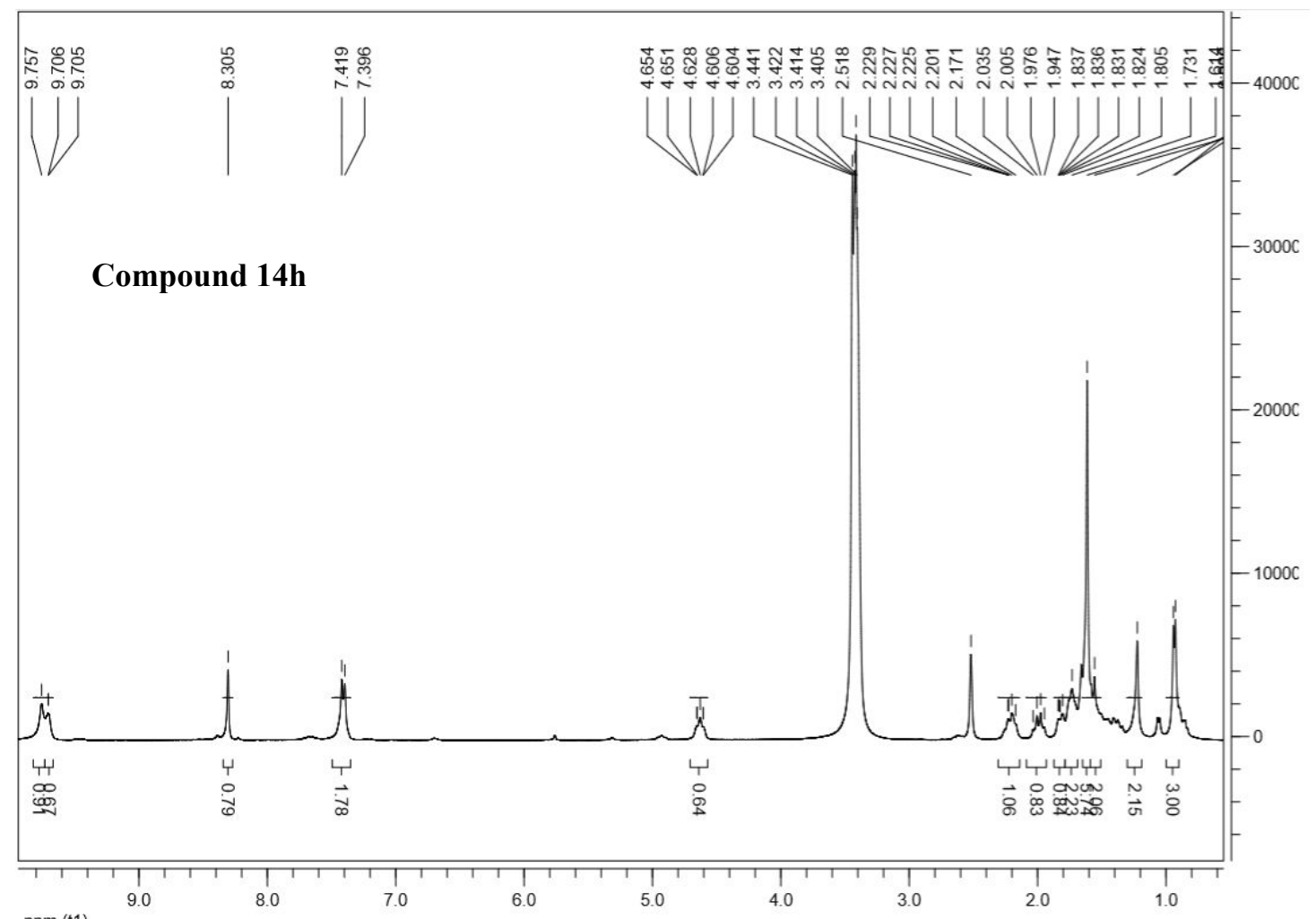

ppm (t1)

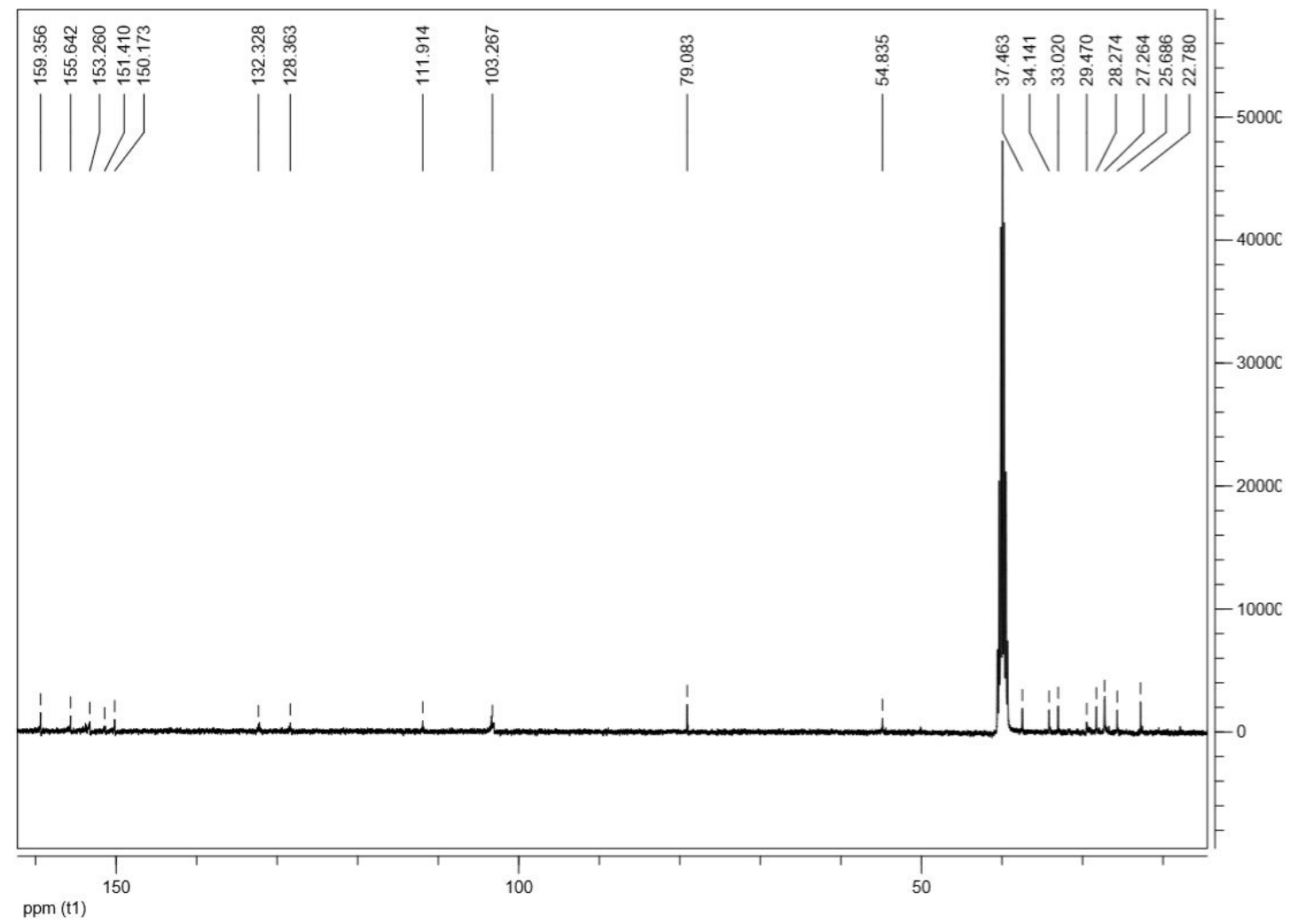




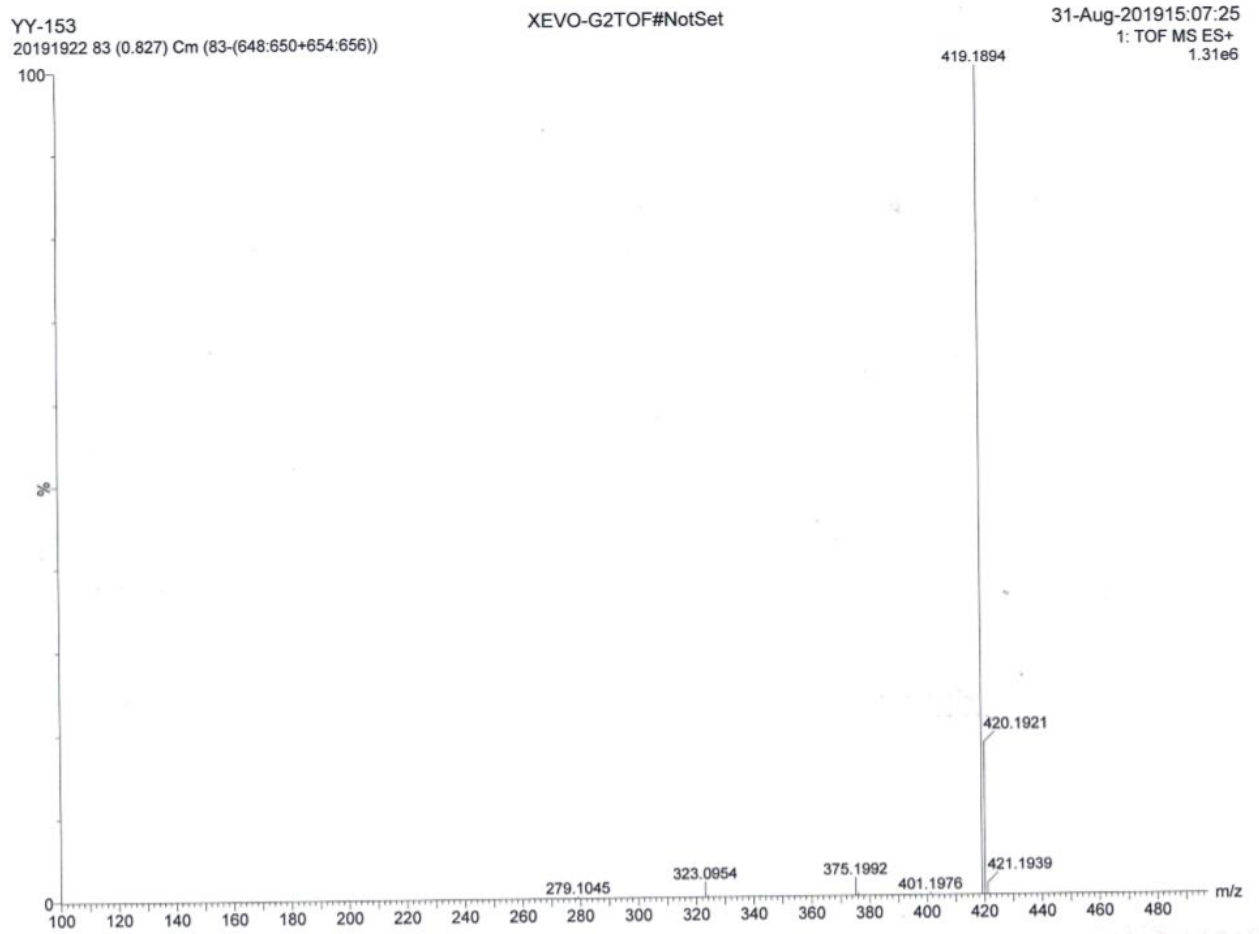




\section{Compound 14i}
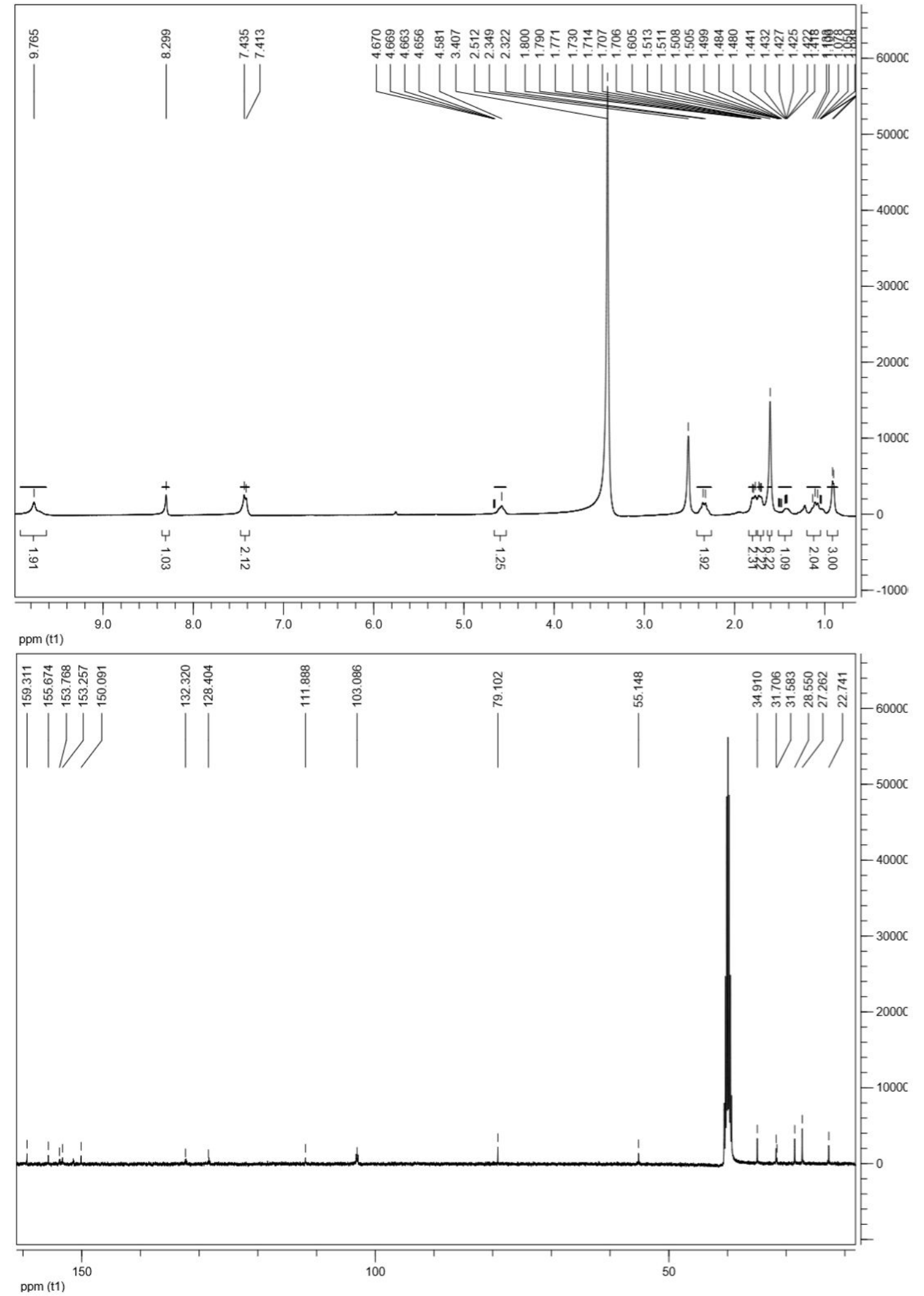


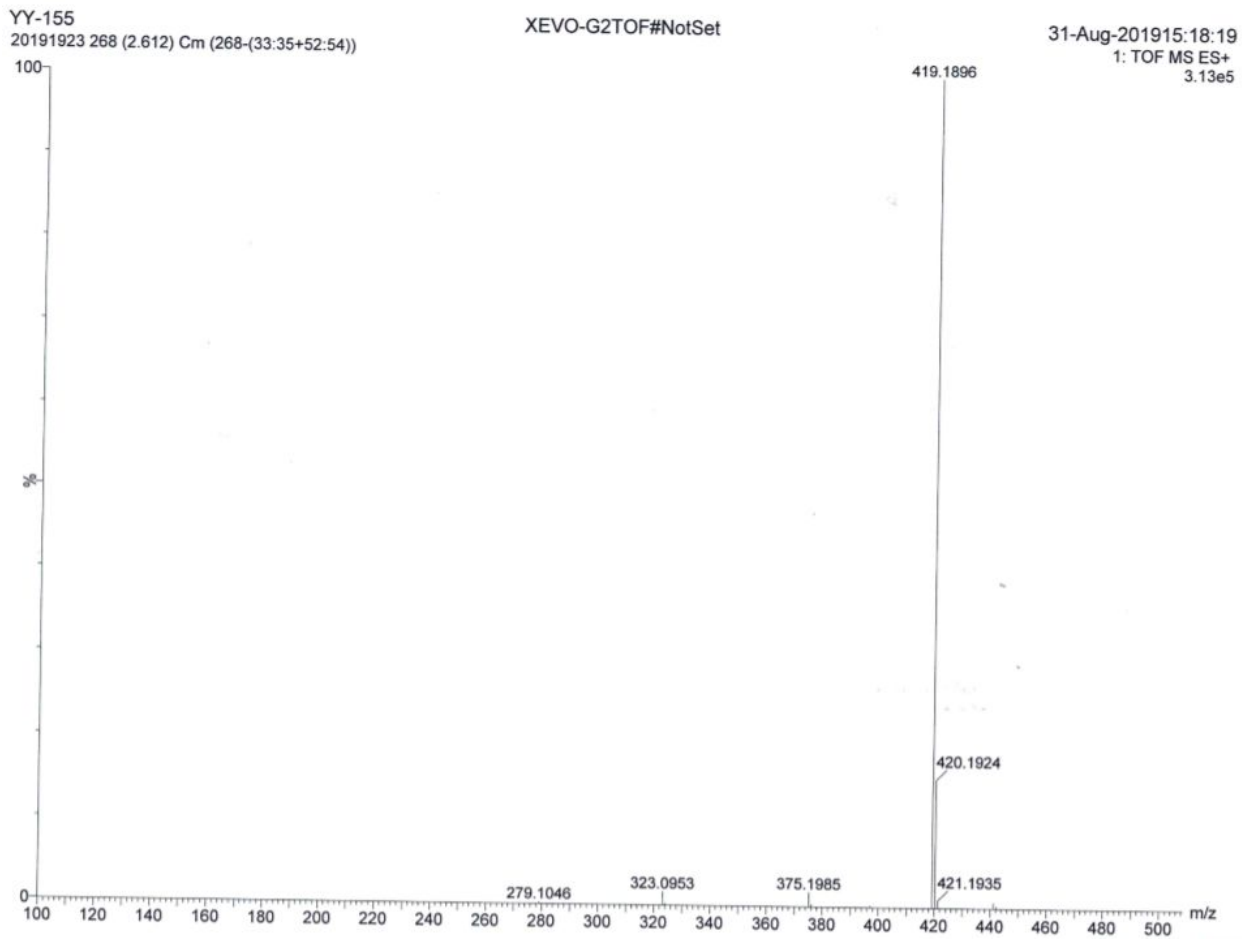




\section{Compound 14j}

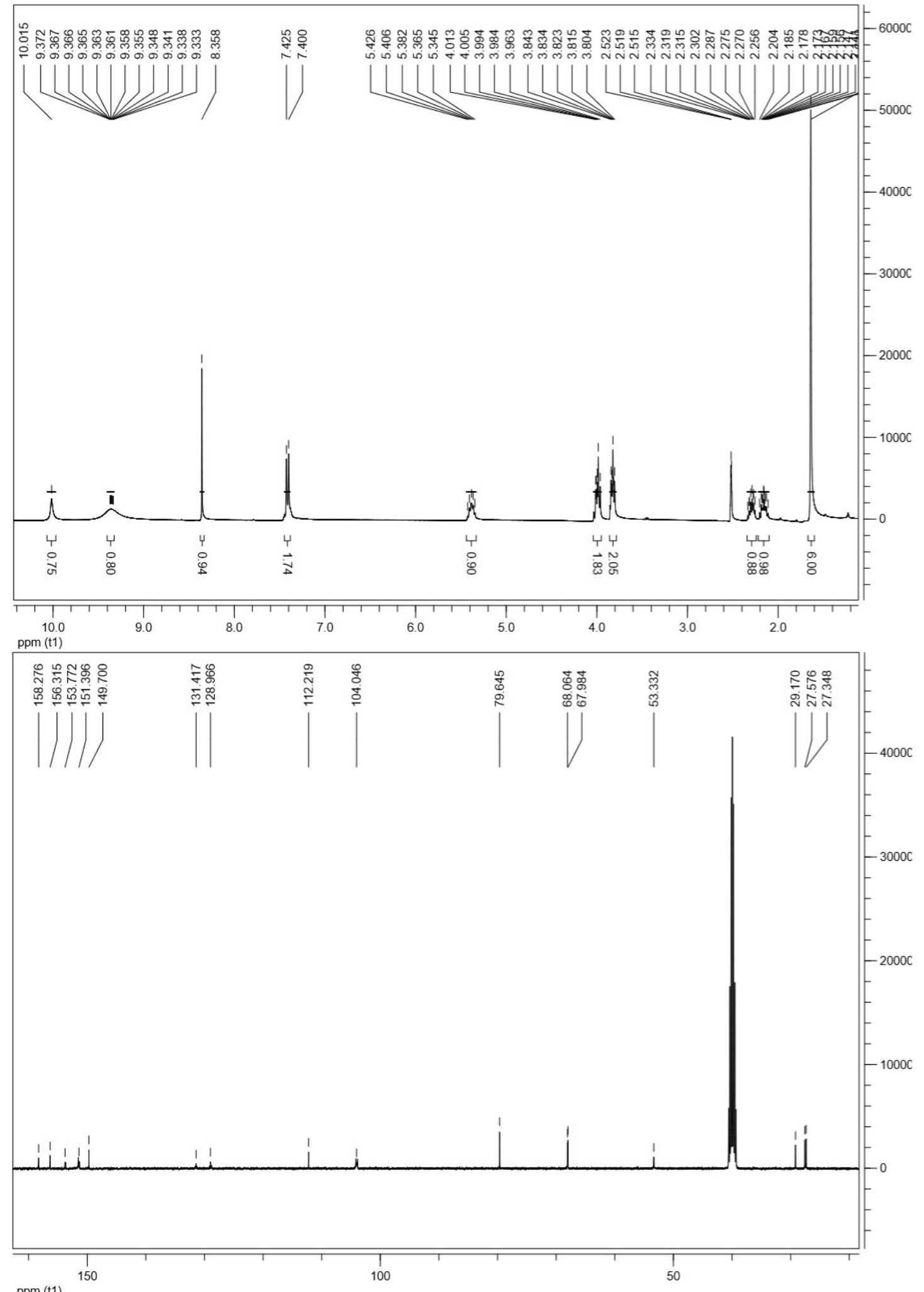




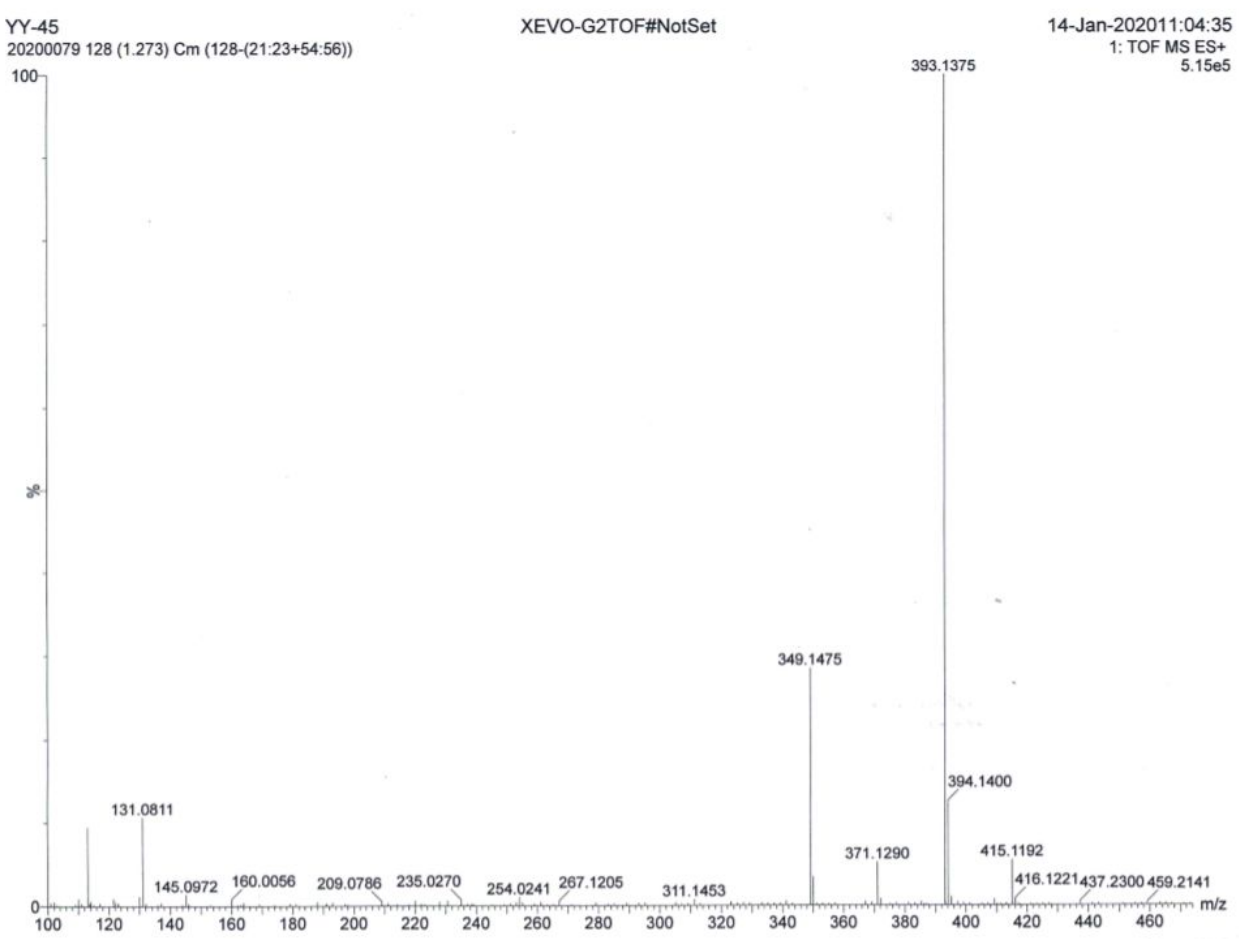




\section{Compound 14k}

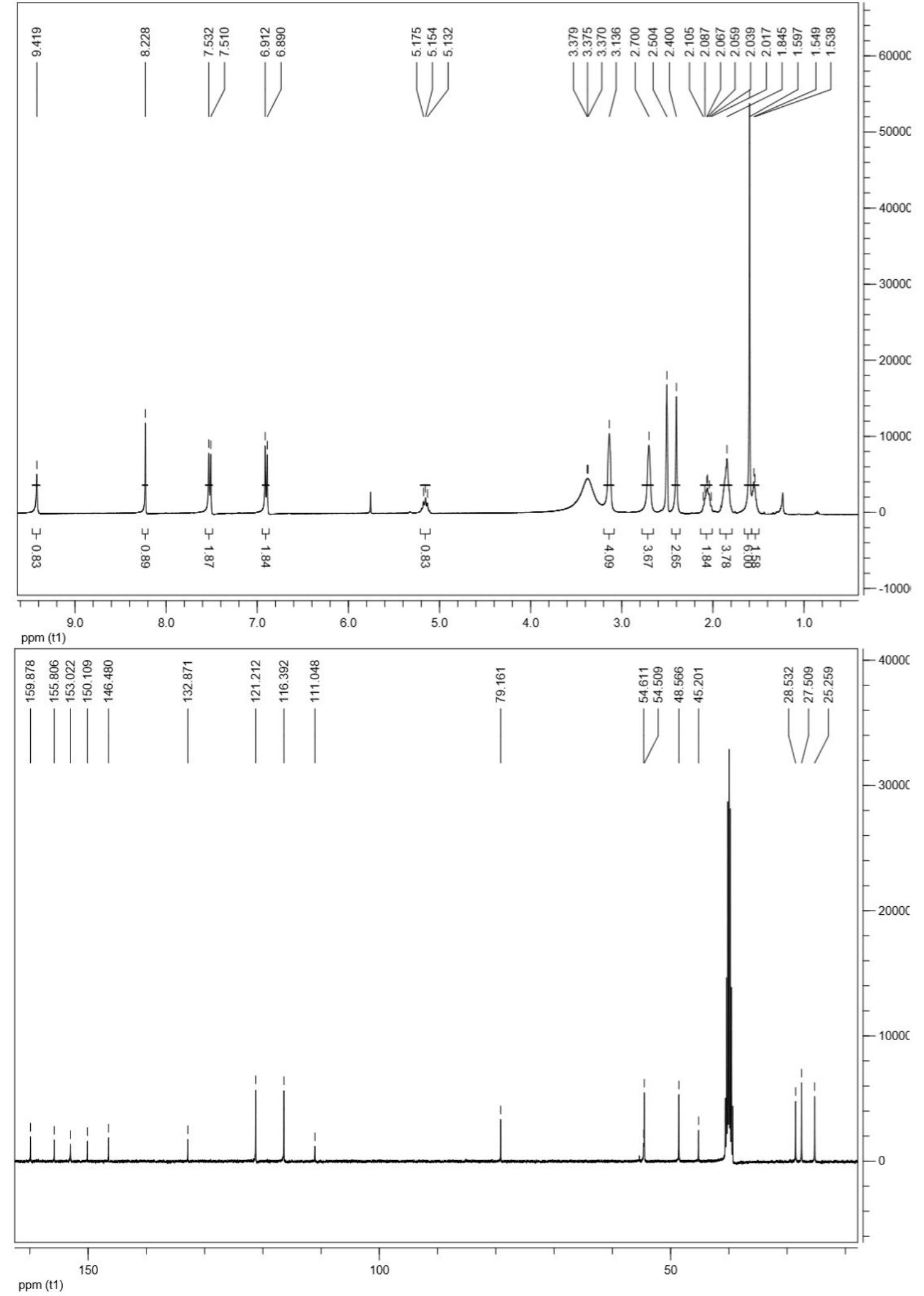




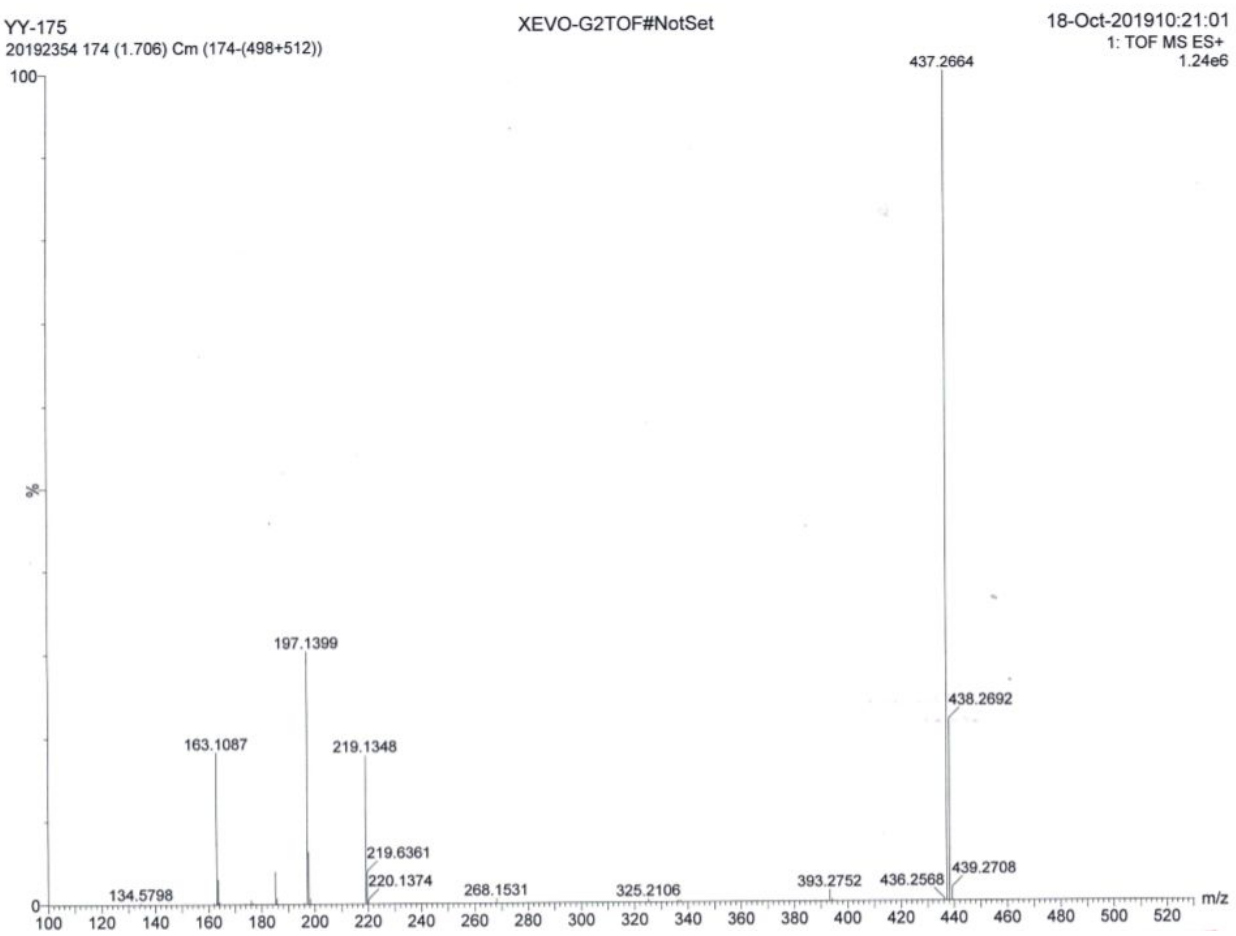




\section{Compound 141}
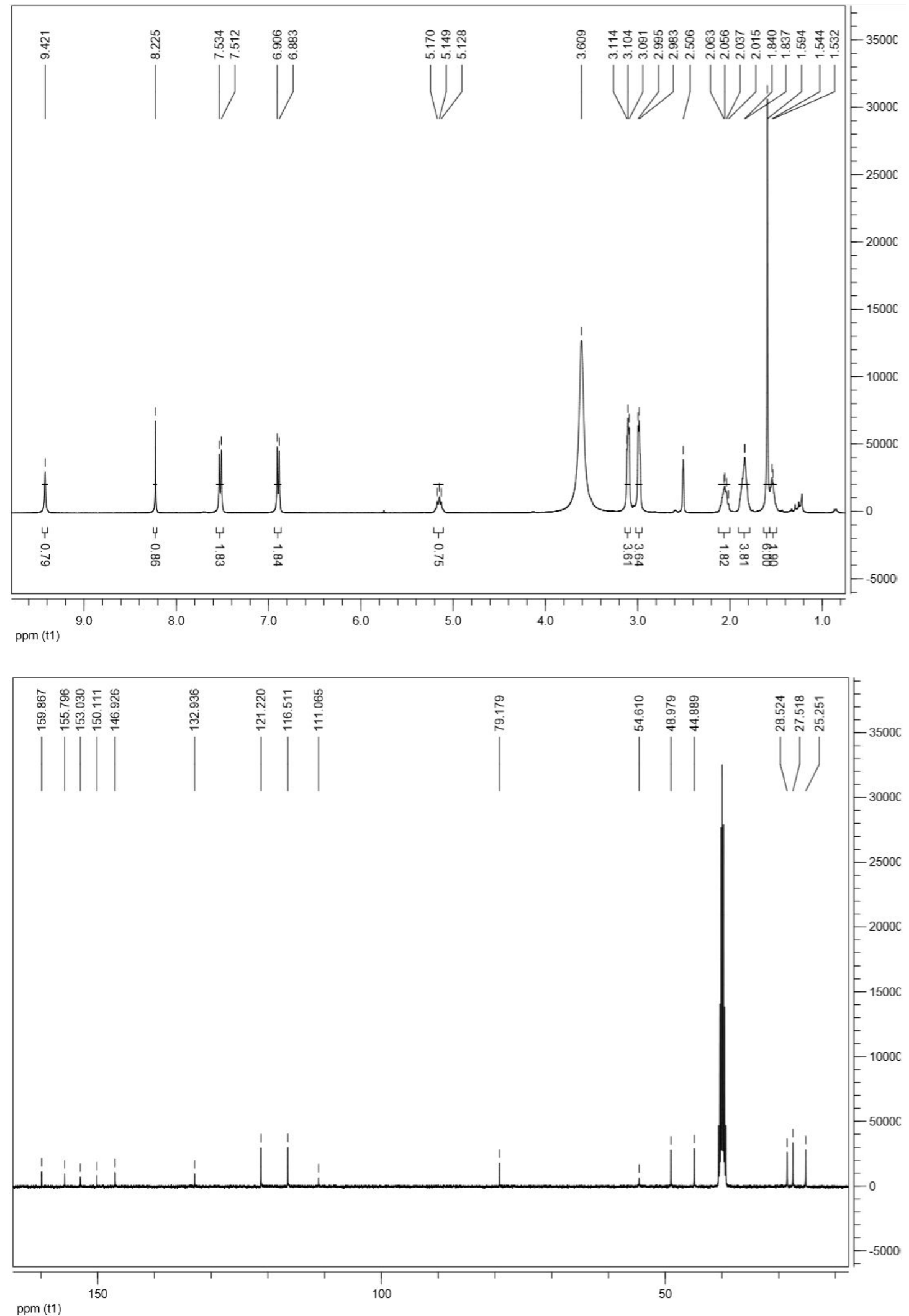


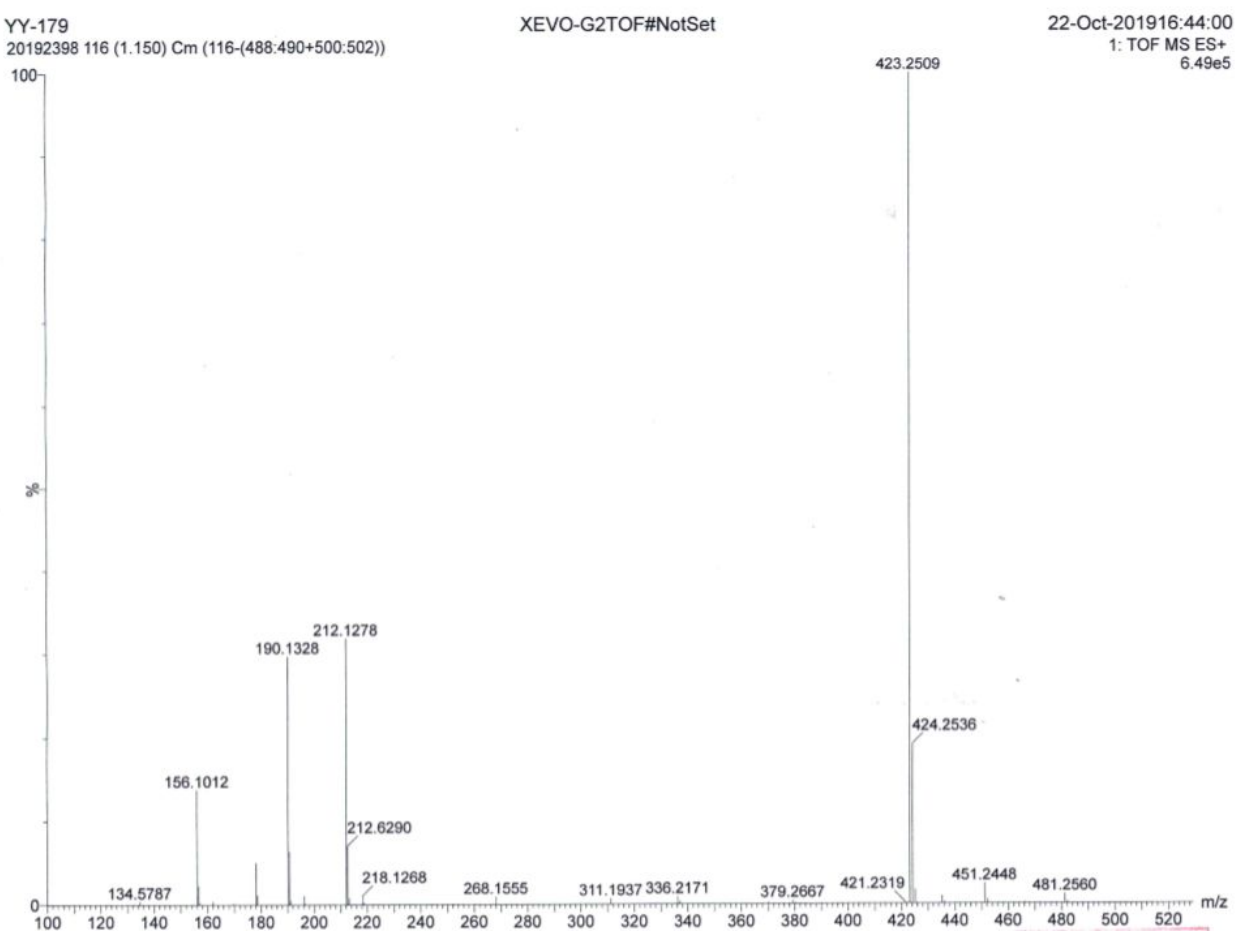




\section{Compound 14m}

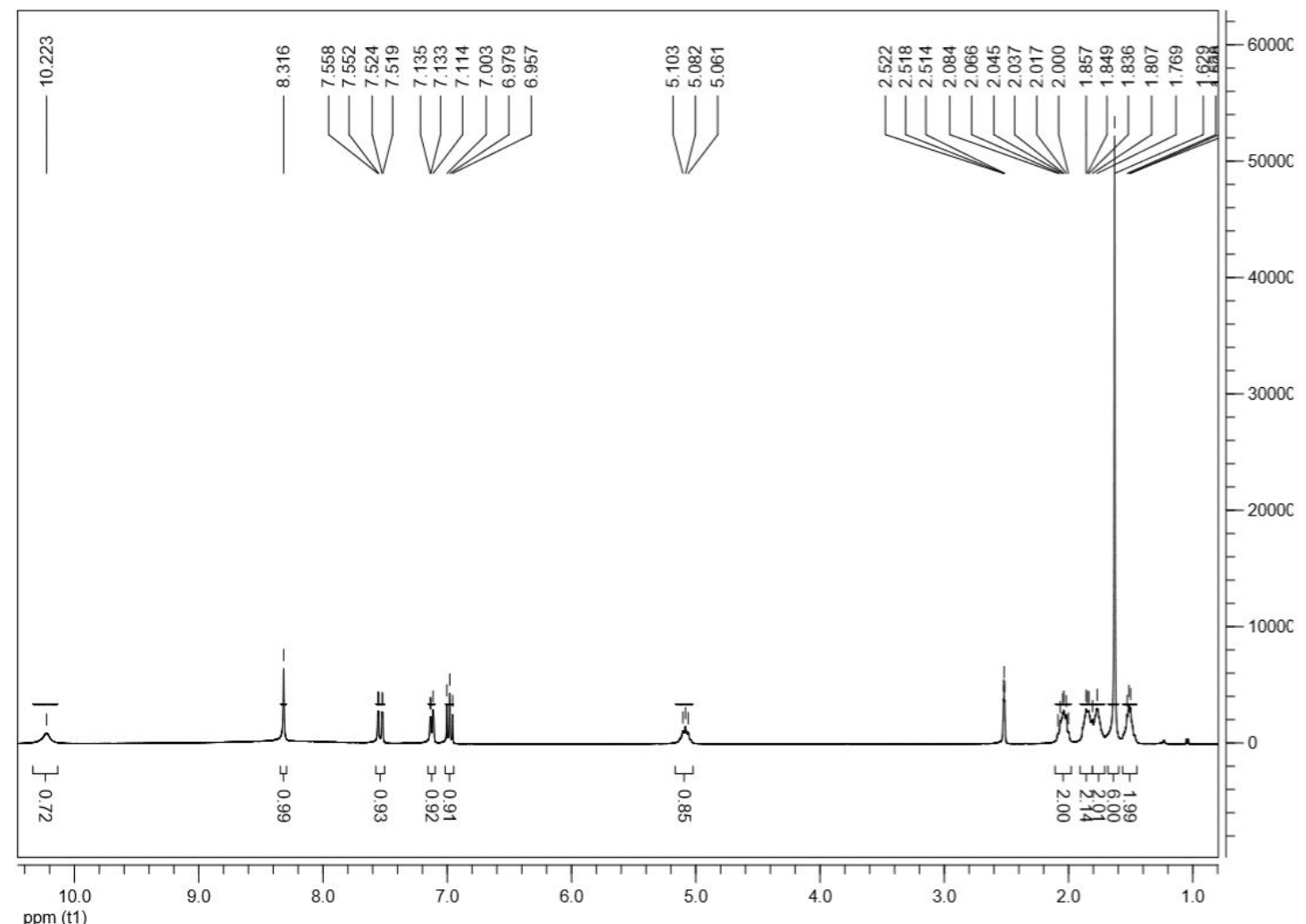

ppm (t1)

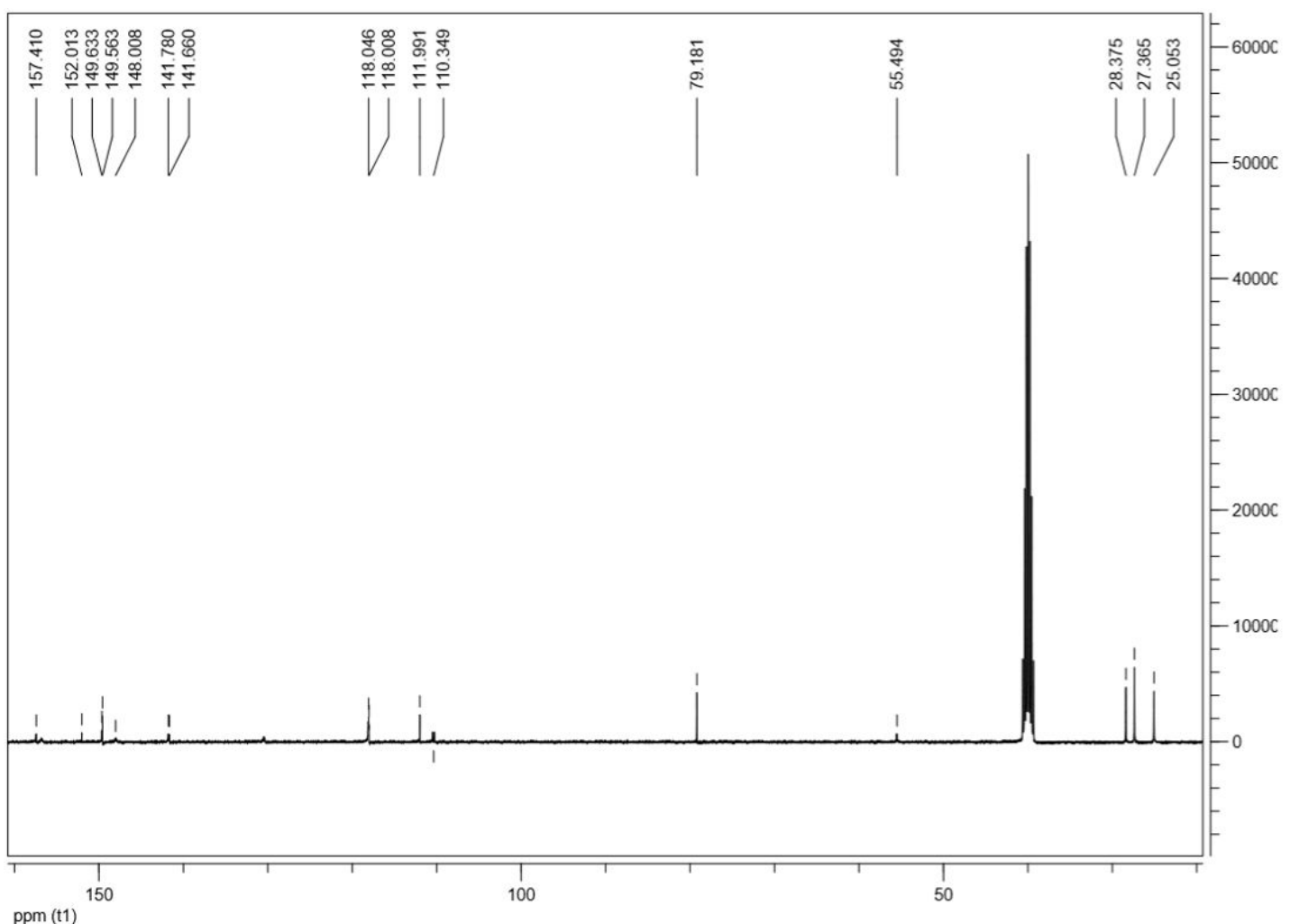




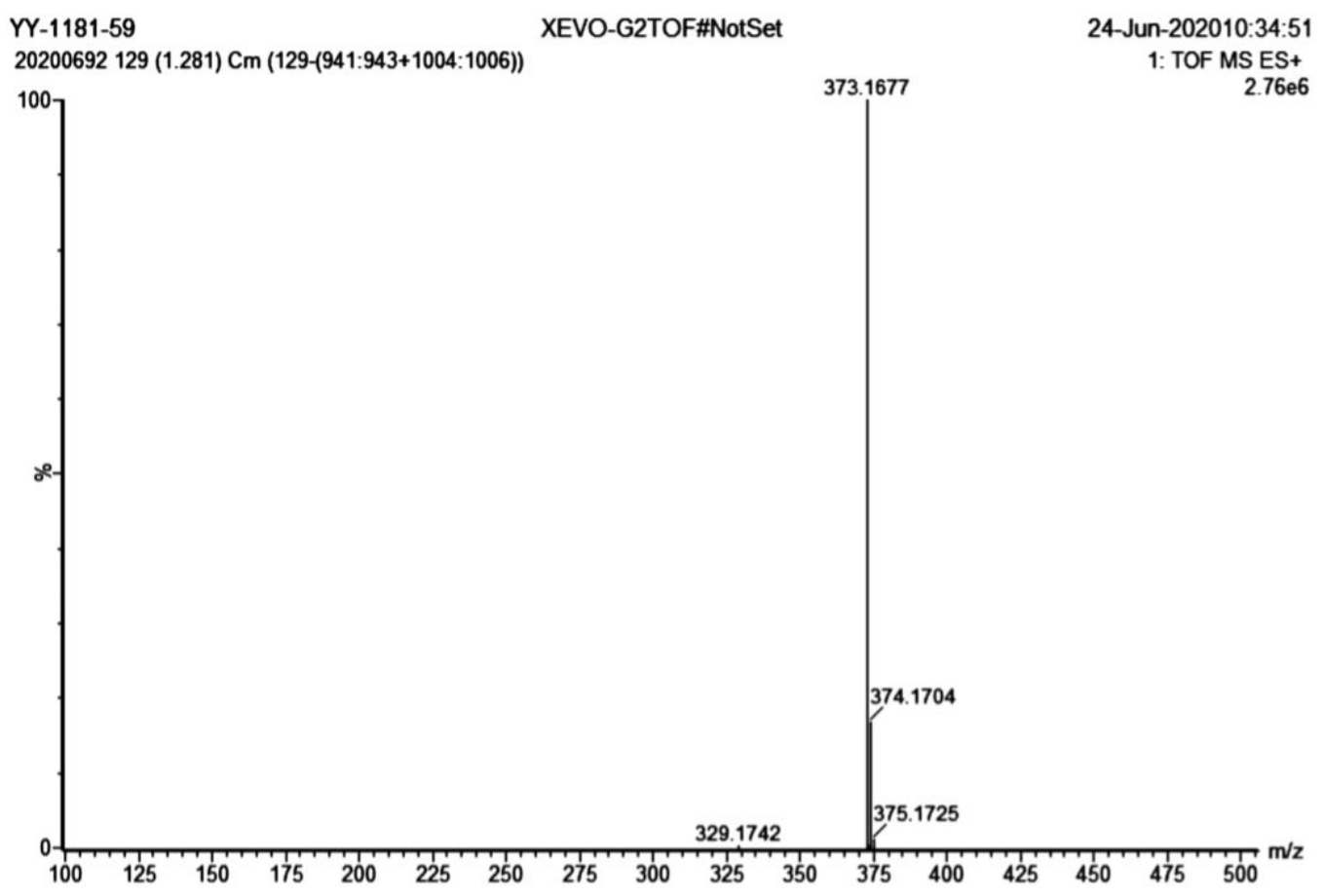




\section{Compound 14n}

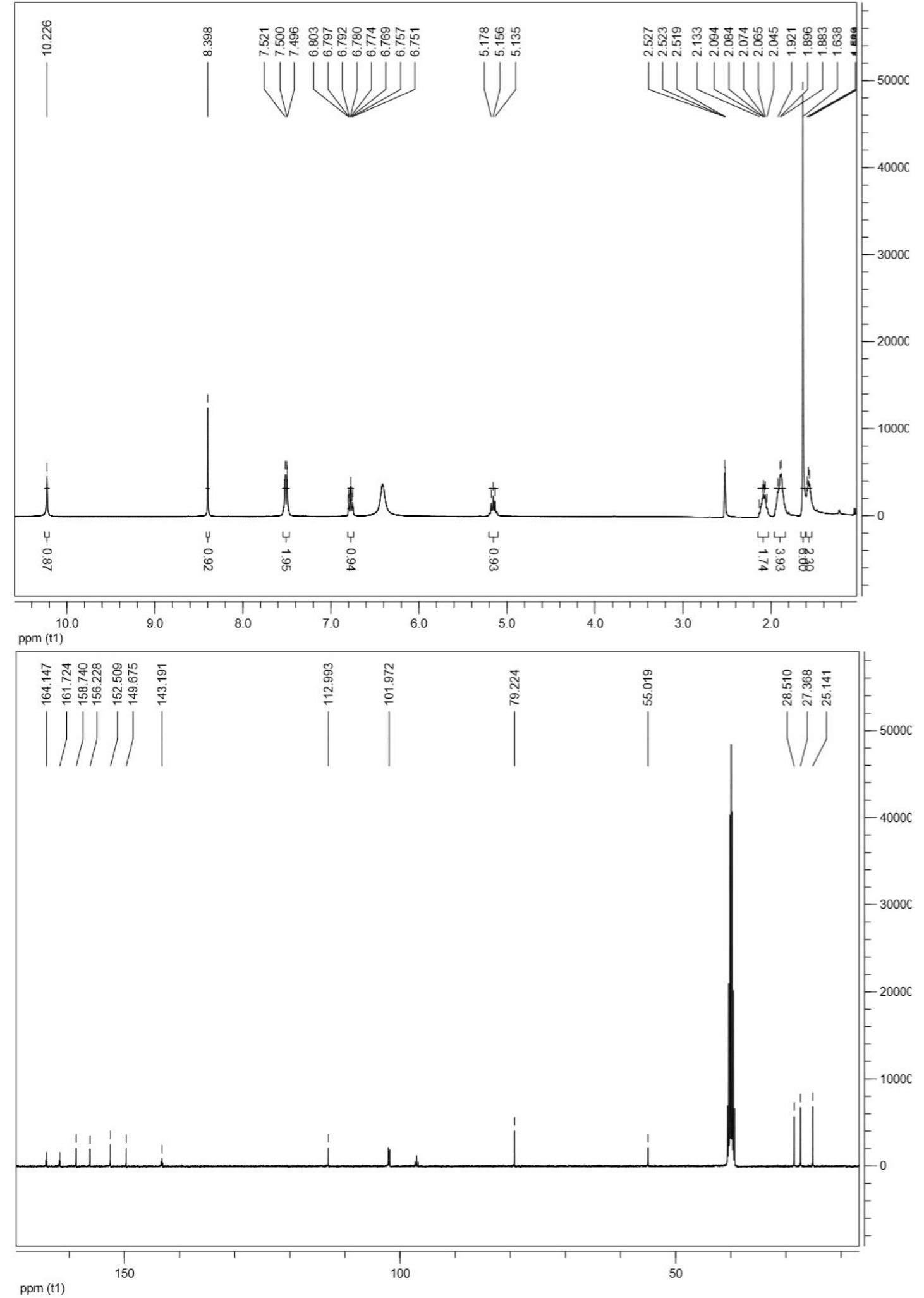




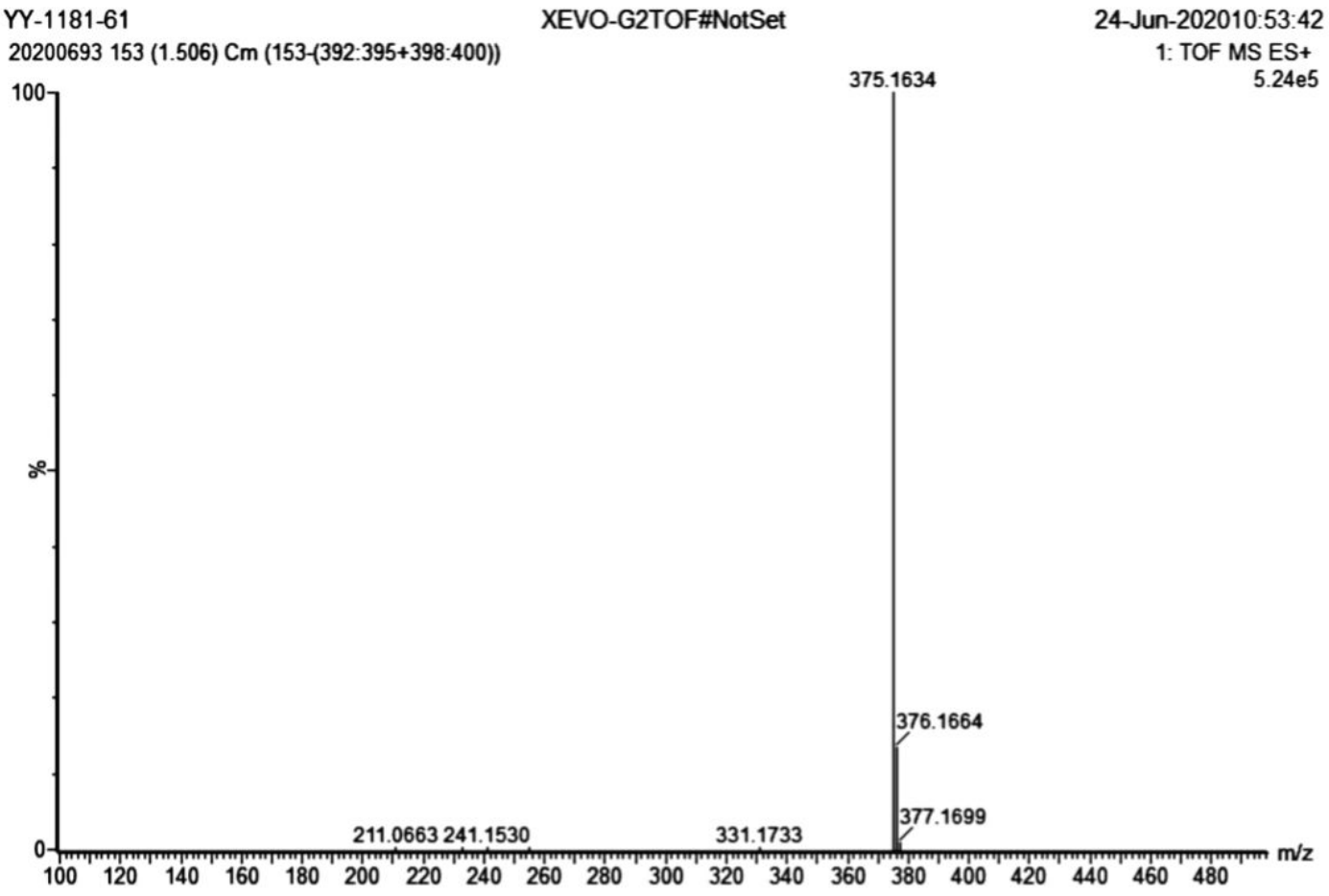




\section{Compound 14o}

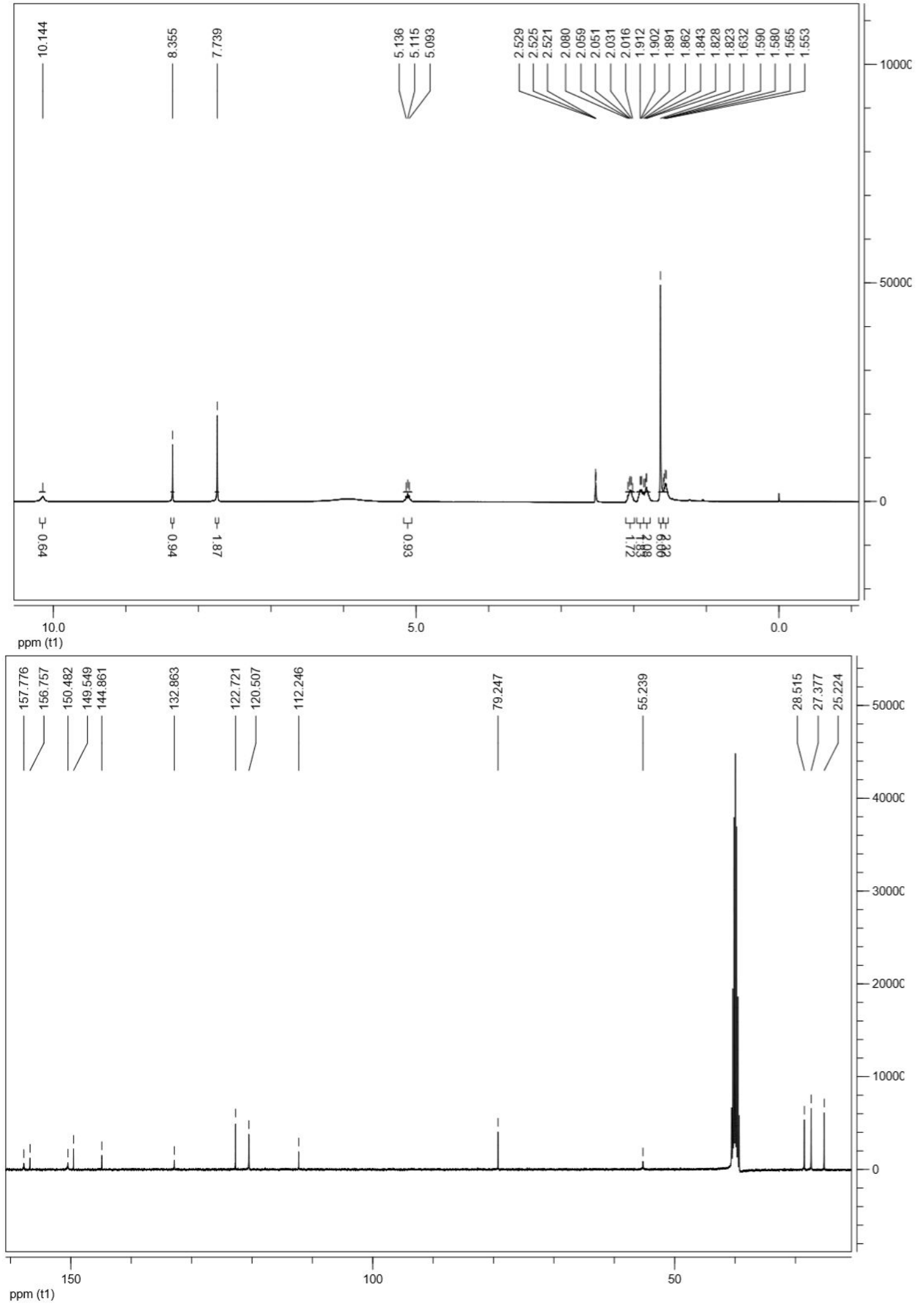


YY-1181-53

20200691109 (1.090) Cm (109-(568:570+576:578))

$\left.{ }^{100}\right]$
XEVO-G2TOF\#NotSet

24-Jun-202010:24:33

1: TOF MS ES+

423.0990

$1.26 \mathrm{e} 6$

ㅇ-

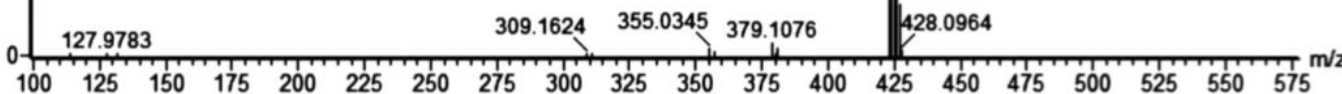




\section{Compound 14p}
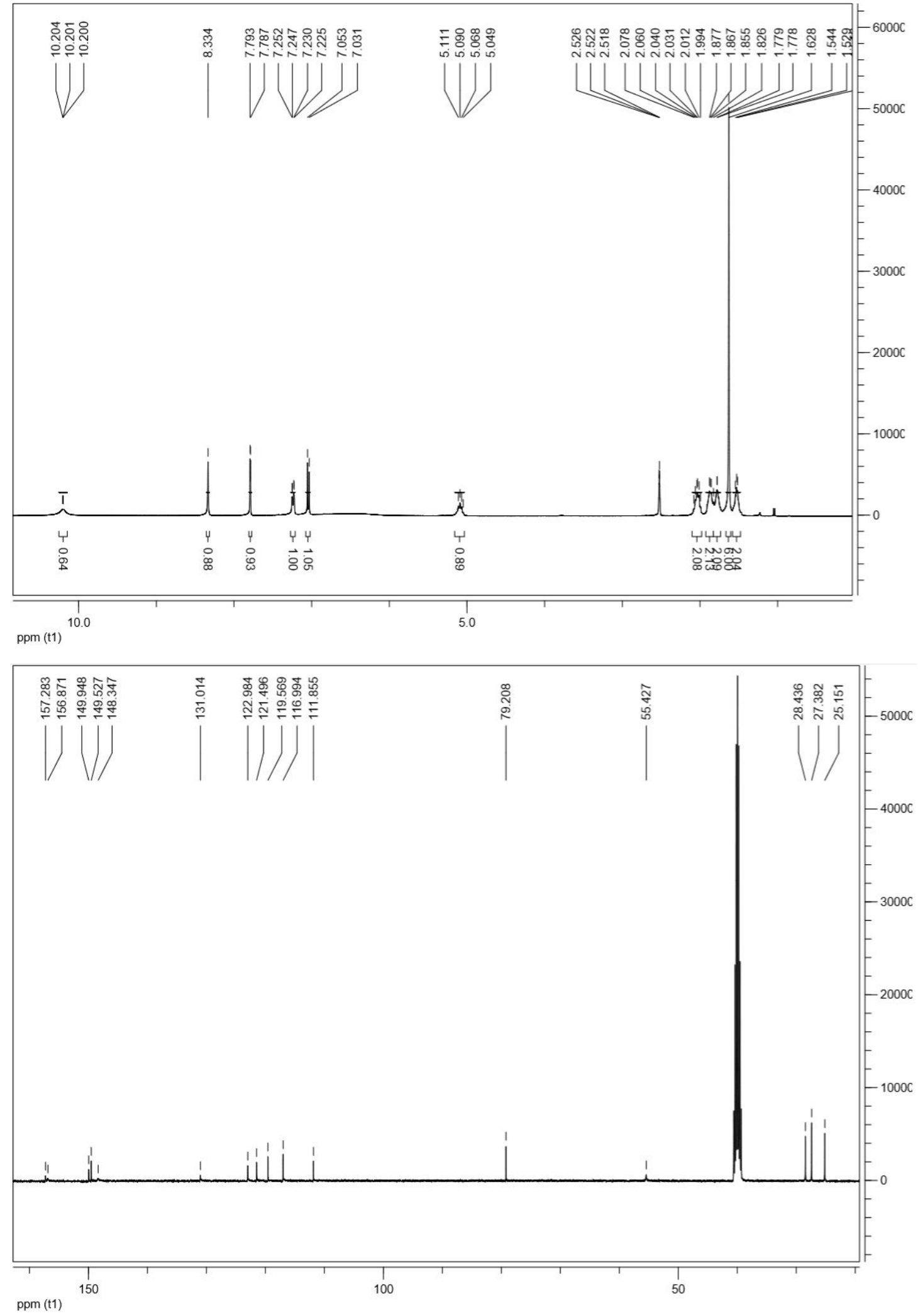


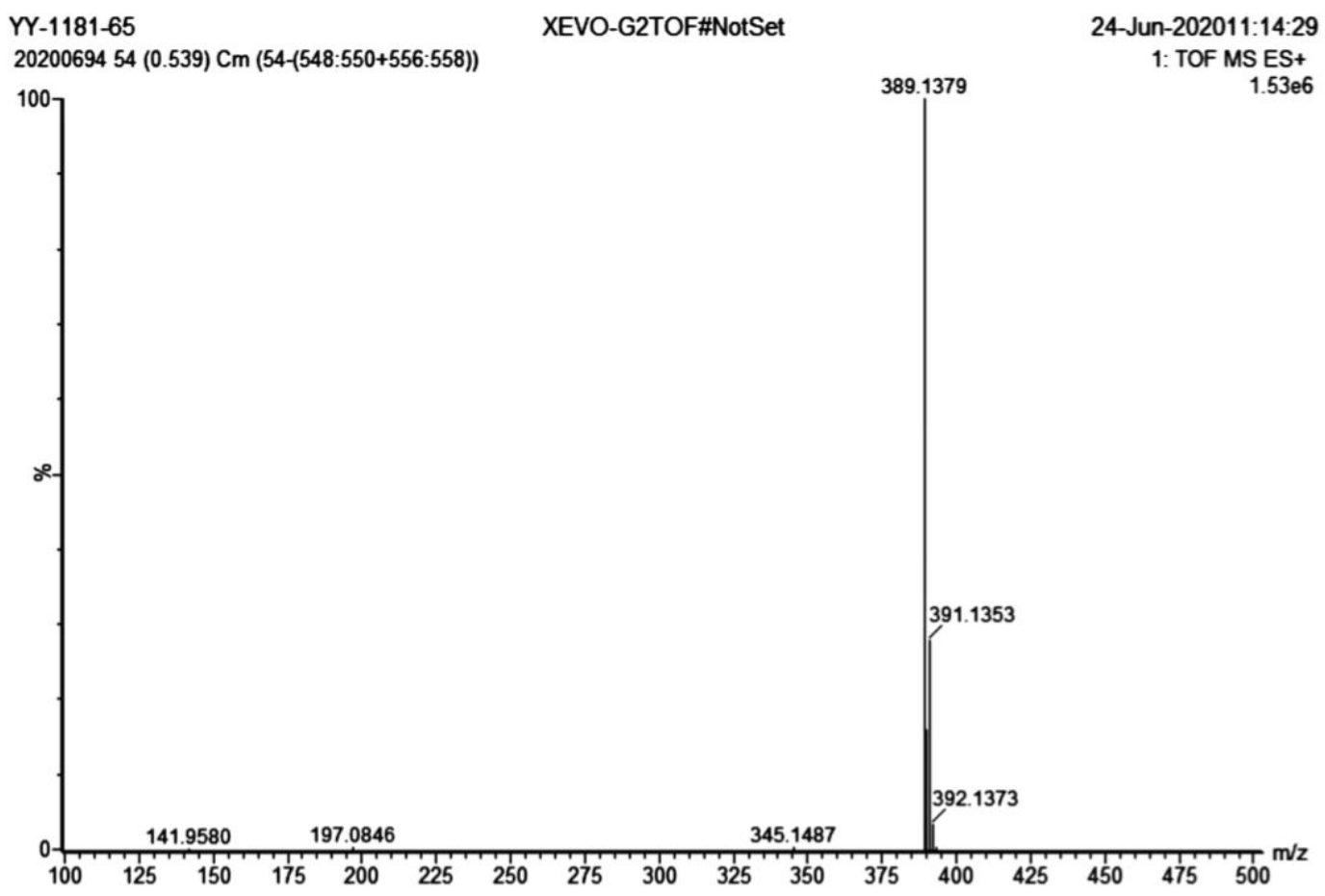




\section{Compound 14q}

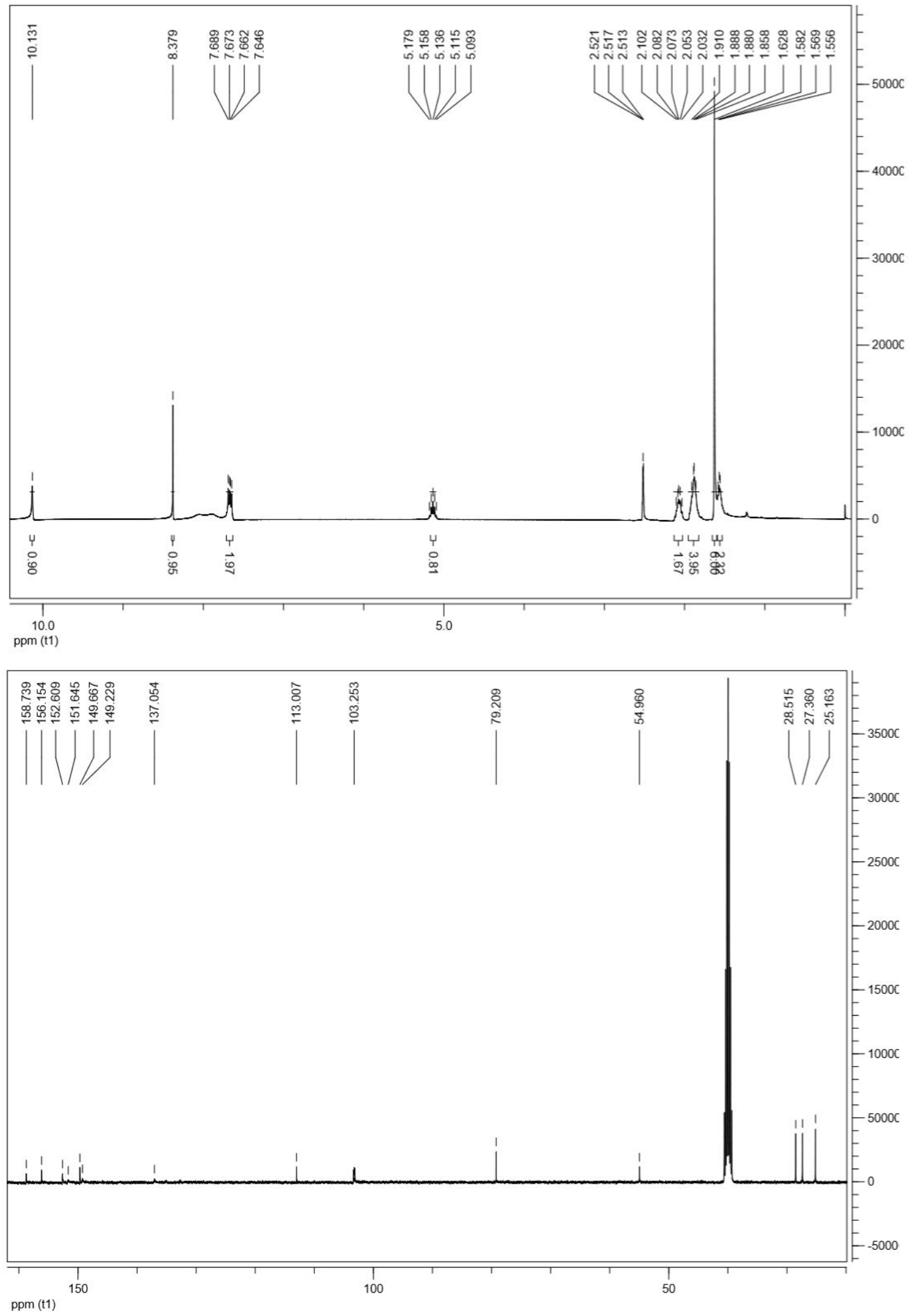




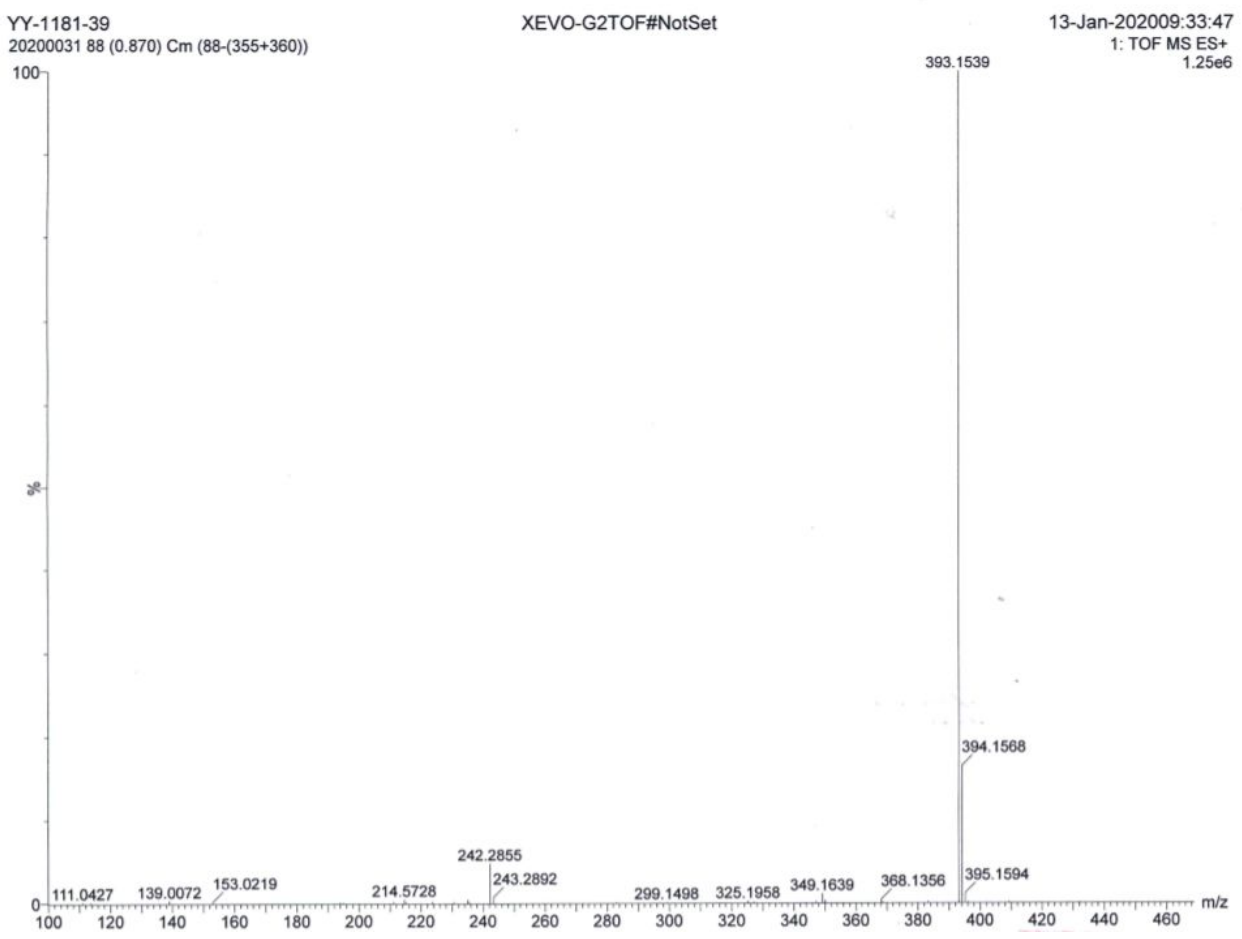




\section{Compound 14r}

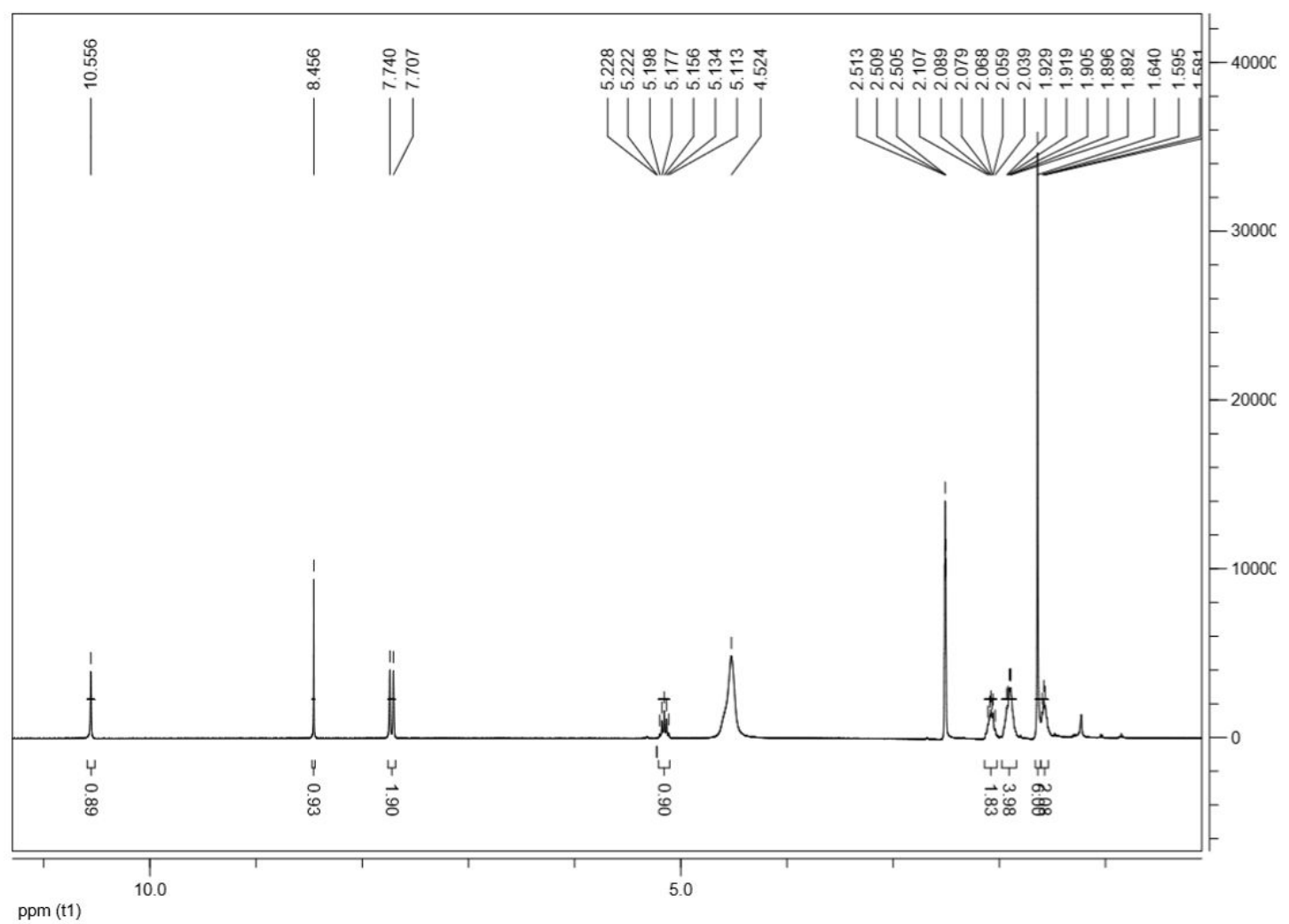

ppm (t1)

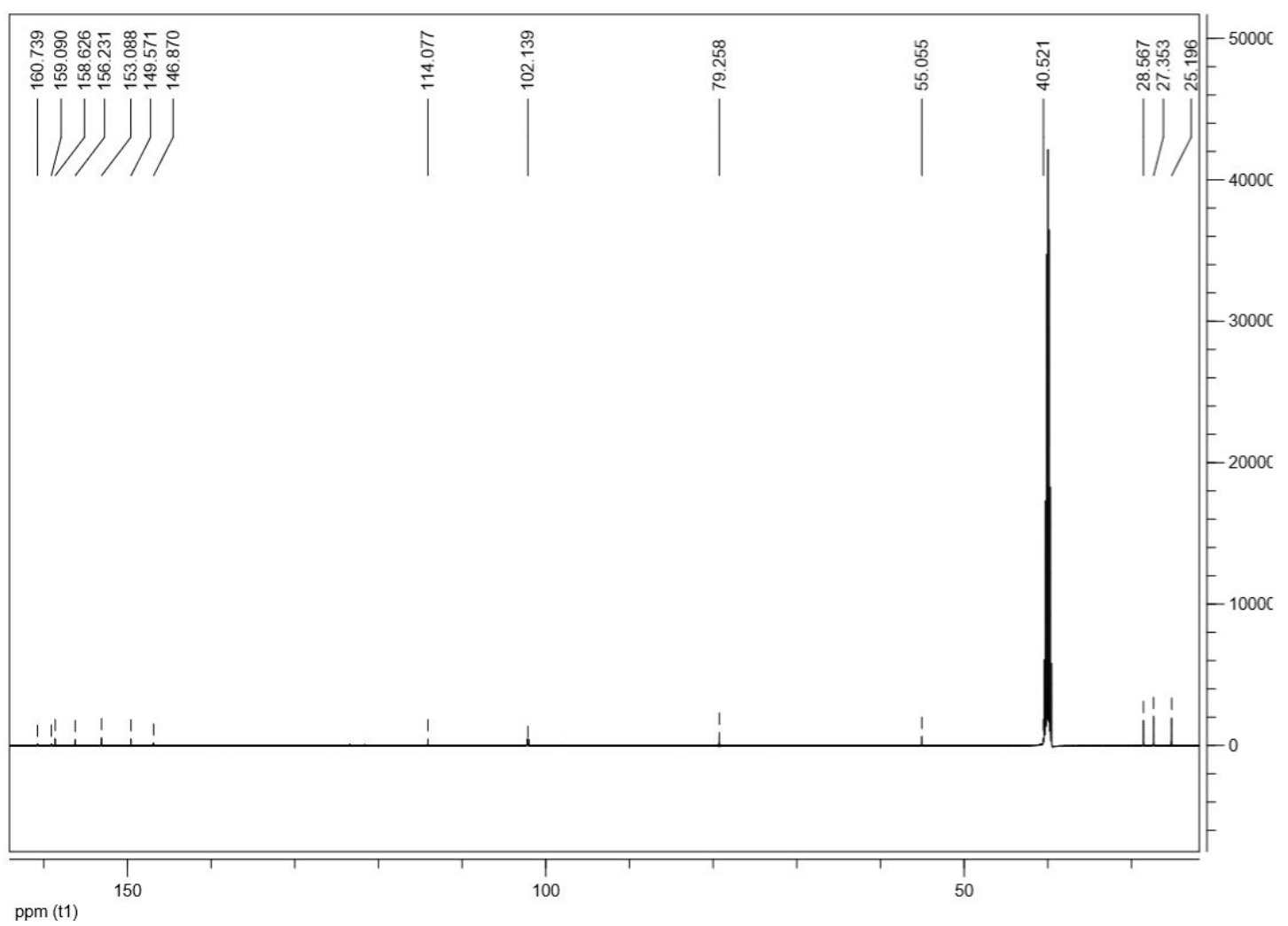




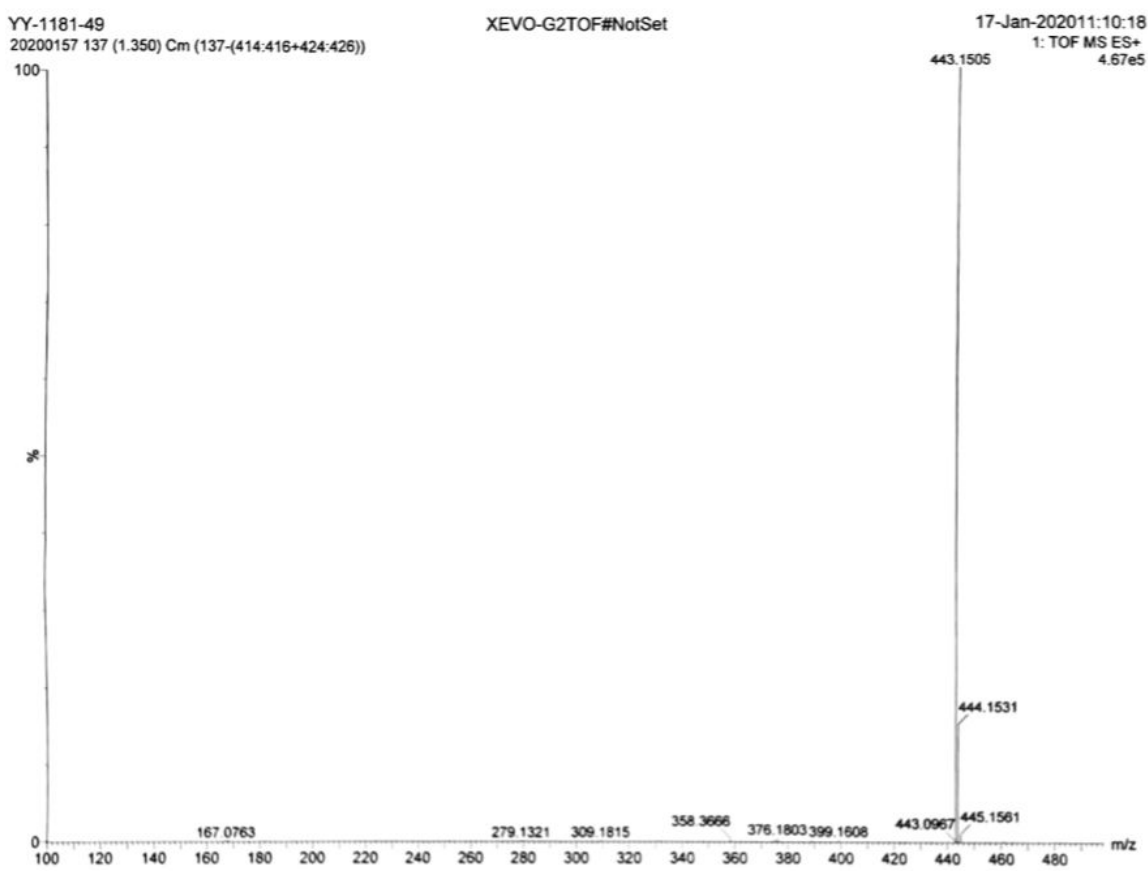




\section{Compound 14s}

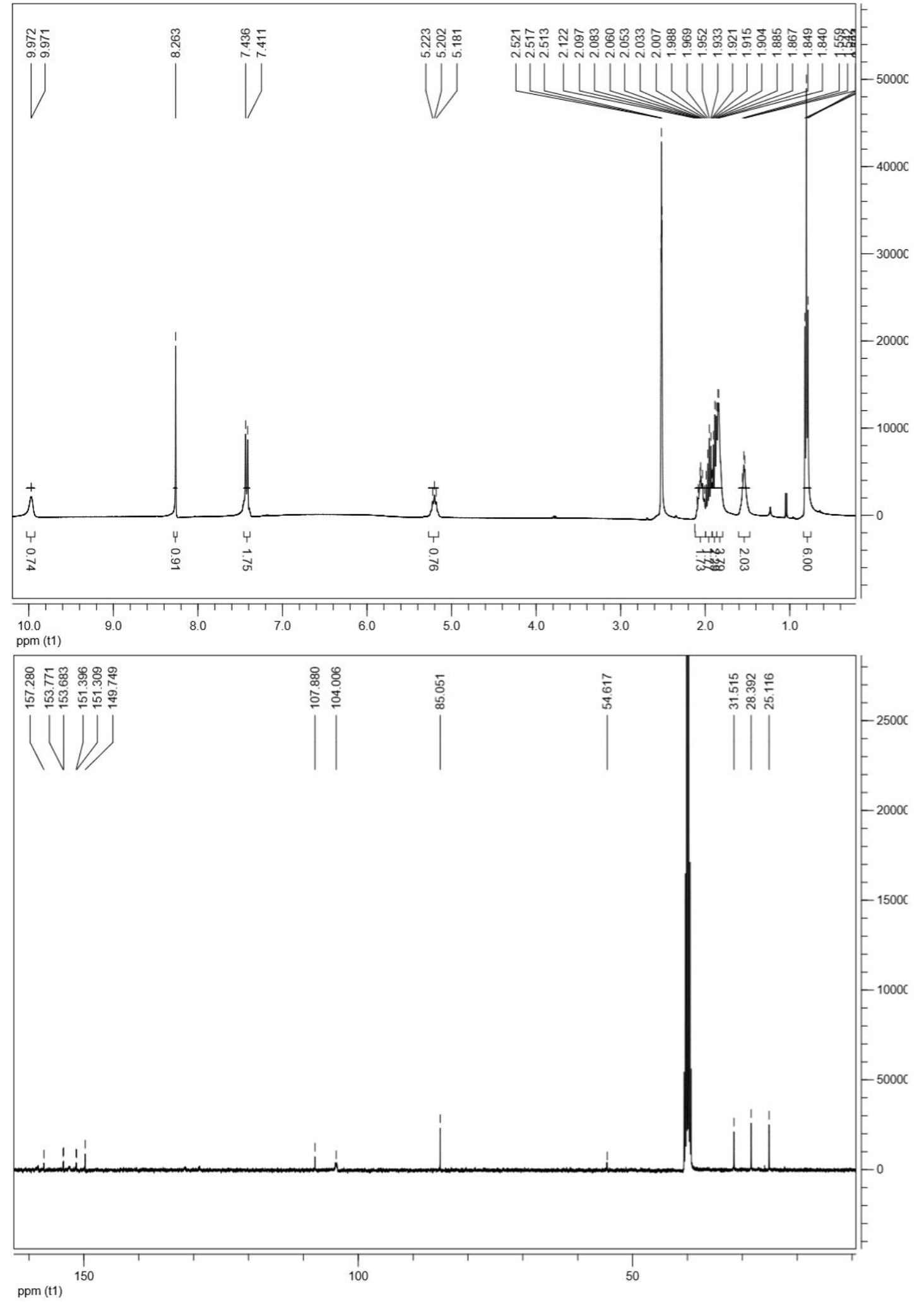




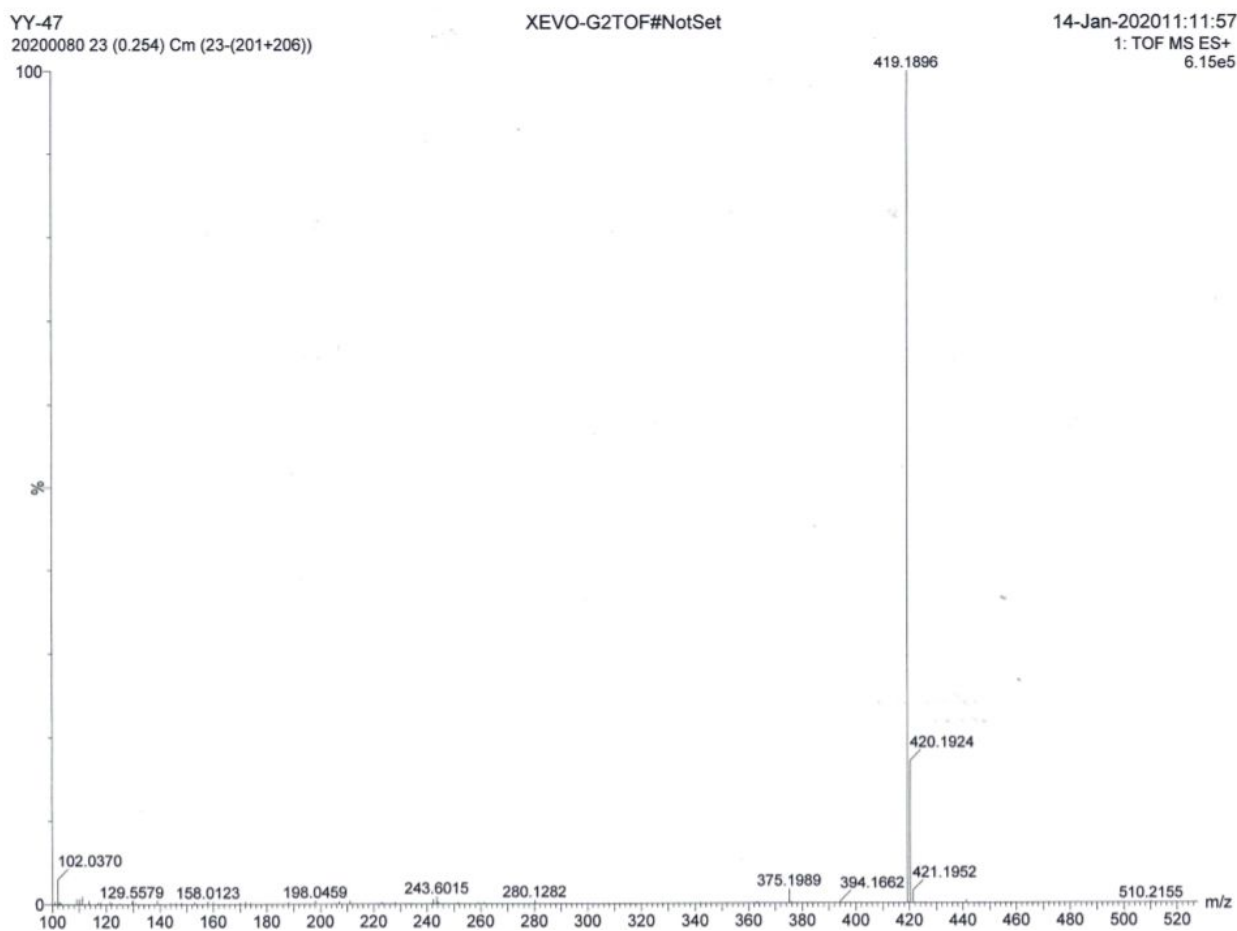


Compound 15a

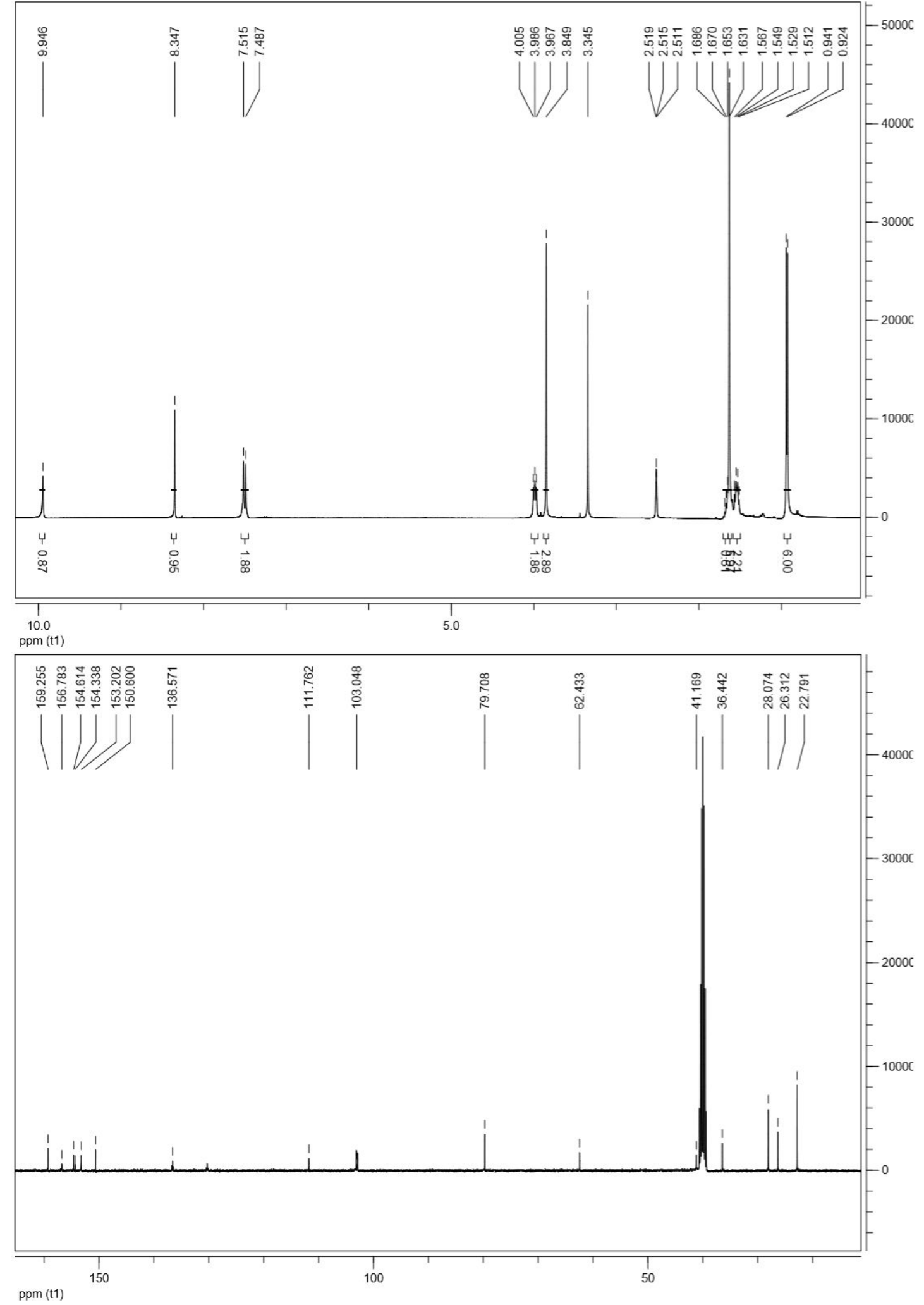




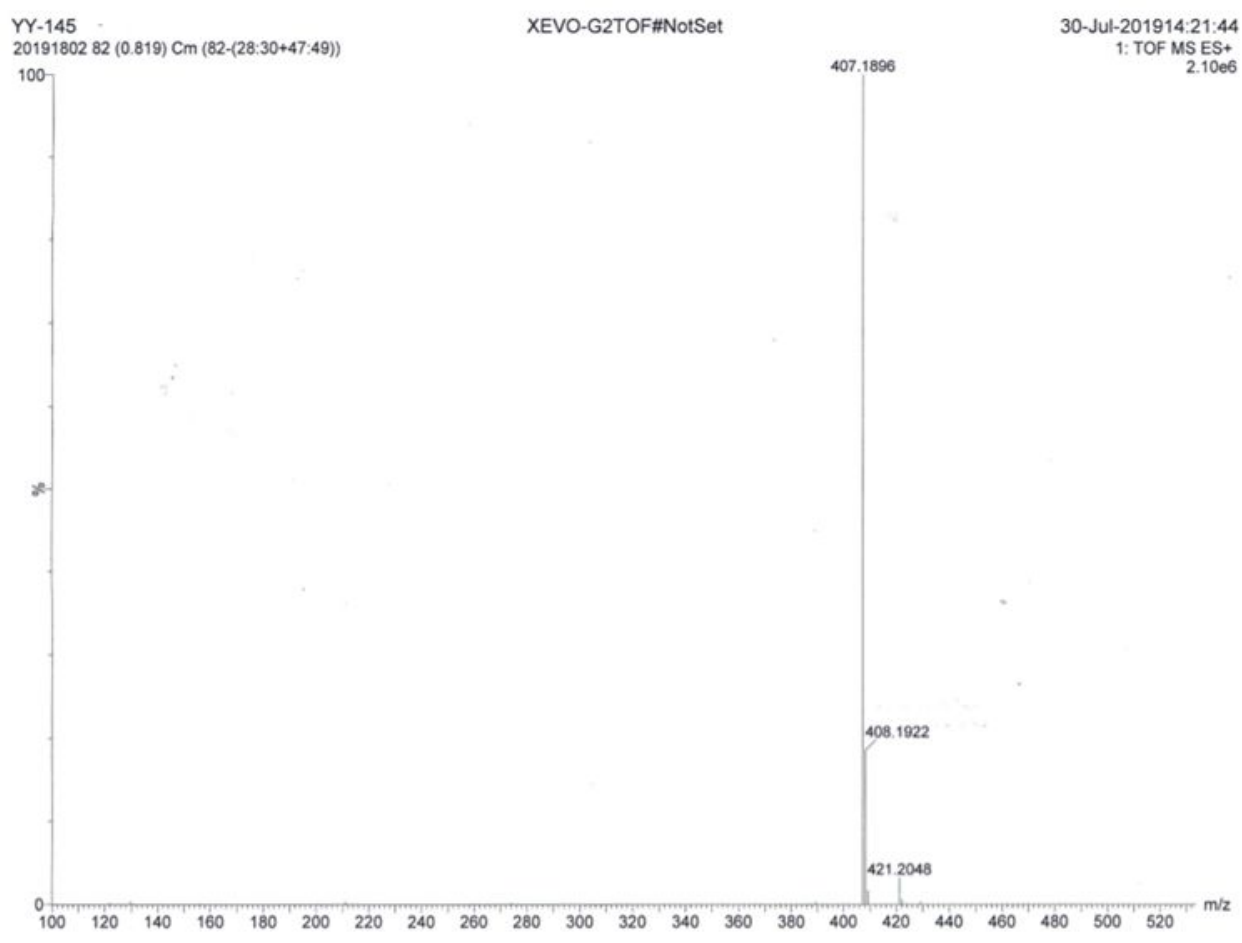

